\title{
The water entry of multi-droplet streams and jets
}

\author{
Nathan B. Speirs ${ }^{1}$, Zhao Pan ${ }^{1}$, Jesse Belden ${ }^{2} \&$ Tadd T. Truscott ${ }^{1 *}$ \\ ${ }^{1}$ Department of Mechanical and Aerospace Engineering, \\ Utah State University, Logan, UT 84322, USA \\ 2 Naval Undersea Warfare Center Division Newport, \\ 1176 Howell Street, Newport, Rhode Island 02841, USA.
}

May 1, 2018

1

\begin{abstract}
Water entry has been studied for over a century, but few studies have focused on multiple droplets impacting on a liquid bath sequentially. We connect multi-droplet streams, jets, and solid objects with physical based scaling arguments that emphasize the intrinsically similar cavities. In particular, the cavities created by the initial impact of both droplet streams and jets on an initially quiescent liquid pool exhibit the same types of cavity seal as hydrophobic spheres at low Bond number, some of which were previously unseen for jets and droplet streams. Low frequency droplet streams exhibit an additional three new cavity seal types unseen for jets or solid spheres that can be predicted with a new non-dimensional frequency. The cavity depth and cavity velocity for both droplet and jet impact are rationalized by an energy scaling analysis and the Bernoulli equation.
\end{abstract}

\section{Introduction}

The water entry of solid bodies and liquid entering liquid, in the form of droplets or continuous jets, has been studied extensively (Worthington \& Cole, 1900 May \& Woodhull, 1948, Engel, 1966; Duclaux et al., 2007, Aristoff \& Bush, 2009: Truscott et al., 2012, Mansoor et al., 2014, Truscott et al., 2014). In some cases, the sub-surface air cavities formed by liquid jets and solid bodies (e.g., spheres) are remarkably similar (Fig. 1k,d). While a single liquid droplet forms a much shallower cavity (Fig. 17), a stream of multiple millimetric sized droplets impacting in rapid succession can form deep, narrow cavities resembling those formed by solid spheres and continuous jets (Fig. 1b). In this paper, we investigate the water entry of such multiple droplet streams, characterizing the cavity physics and drawing comparisons with jet and sphere water entry.

${ }^{1}$ correspondence: taddtruscott@gmail.com 


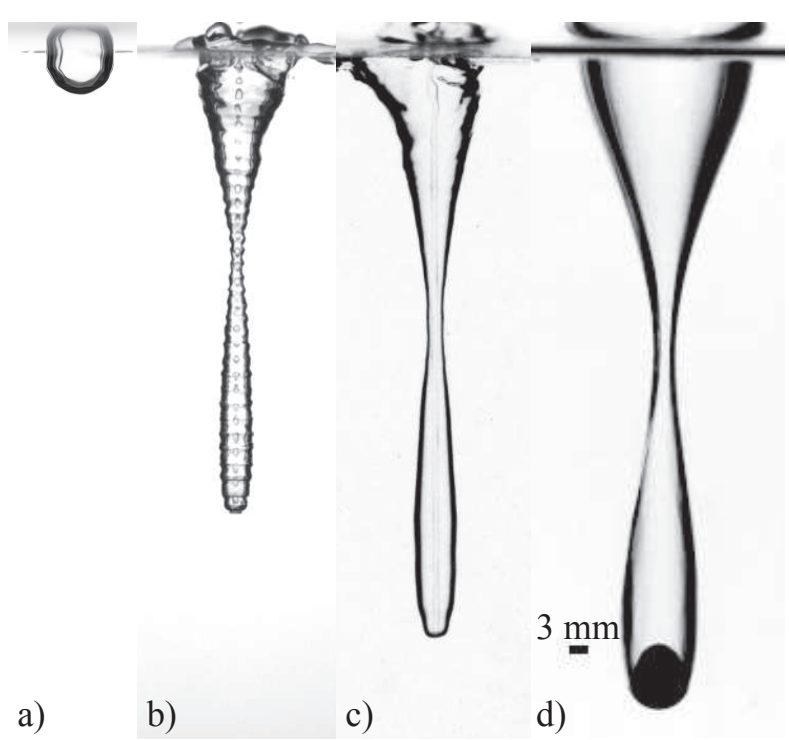

Figure 1: Images of several cavity shapes created by various impacting bodies. a) Maximum cavity size from a single droplet impacting a pool of water $(d=3.39$ $\mathrm{mm}, U=2.14 \mathrm{~m} / \mathrm{s})$. Cavity shape just prior to pinch off for cavities created by: b) a multi-droplet stream $\left.\left(d=1.53 \mathrm{~mm}, f=2500 \mathrm{~Hz}, U_{s}=6.17 \mathrm{~m} / \mathrm{s}\right), \mathrm{c}\right)$ a jet $\left(d=1.17 \mathrm{~mm}, U_{s}=6.07 \mathrm{~m} / \mathrm{s}\right)$, and d) a hydrophobic sphere $(d=9.53 \mathrm{~mm}$, $U=2.43 \mathrm{~m} / \mathrm{s})$. Scale bar applies to all images. 
Prior research on liquid and solid body water entry has shown the cavity dynamics may be influenced by inertial, gravitational, viscous and/or surface tension effects depending on the scales involved in the problem (Truscott et al. 2014). The relative importance of these effects are characterized by the Bond number $\left(B o=\rho g L^{2} / \sigma\right)$, the Weber number $\left(W e=\rho U^{2} L / \sigma\right)$, the Froude number $\left(F r=U^{2} / g L\right)$, and the Reynolds number $(R e=\rho U L / \mu)$, where $\rho$ is the fluid density, $g$ is the acceleration of gravity, $\sigma$ is the surface tension, $L$ is a length scale (specified for our study in ??), $U$ is the impact velocity, and $\mu$ is the fluid dynamic viscosity.

Previous studies on single droplet impact inform our study on multi-droplet cavity formation. Research on single droplets impacting a deep pool has found several areas of focus, with the objective to understand the underwater sound of rain (Franz, 1959, Oguz \& Prosperetti, 1990), spray creation (Yarin, 2006), and near surface air entrainment (Thoroddsen et al. . 2003). Engel (1966) and Engel (1967) studied the cavity formation of large droplets (droplet diameter $d_{d}=4.5 \mathrm{~mm}$ ) impacting at high velocities (above $9 \mathrm{~m} / \mathrm{s}$ ) and predicted the cavity depth by equating the kinetic energy of the droplet to the potential energy of the cavity formed. Rodriguez \& Mesler (1988) found that the fall height and shape of the droplet at impact affect the cavity shape. Leng (2001), Morton et al. (2000), Ray et al. (2015) and Cole (2007) characterized a range of cavity behaviors including jet formation, vortex ring formation, coalescence, bubble entertainment and splash dome-over, using $W e$ and $F r$ to classify the physics.

The cavity physics associated with liquid jets impacting liquid pools are quite different from single droplet impact. When a continuous jet of water impacts normal to an initially quiescent free-surface, the impact forms a cavity that is driven deeper into the pool by the jet. The evolution of this cavity can include many features of rigid body impact as indicated in Fig. 1 1 \& \&d. The current study focuses on the initial impact of a jet onto a quiescent free surface up until the cavity collapses. Several common topics persist in studies on cavity forming jet impacts. Cavity dimensions including the final depth of the cavity, the depth of pinch-off and the cavity radius are most prominently discussed by Oguz et al. (1995) and Zhu et al. (2000). They find that the final cavity depth and the pinch-off depth are functions of the Froude number and that the cavity radius is approximately twice the jet radius. Most studies agree that the downward cavity velocity is equal one half the jet velocity (Birkhoff \& Zarantonello, 1957 Oguz et al., 1992, 1995: Zhu et al., 2000, Qu et al., 2013) but Kersten et al. (2003) and Soh et al. (2005) argue to the contrary. A few studies also discuss the motion and deformation of the jet fluid after impact showing that it coats the surface of the cavity (Kersten et al., 2003; Zhu et al., 2000, Oguz et al., 1995. Soh et al. 2005). It is worth noting that following the transients associated with jet impact onto a quiescent pool, steady-state air entrainment may occur at later times if the jet is allowed to continuously flow into the pool (Lorenceau et al. 2004, Kiger \& Duncan, 2012).

Bick et al. (2010) performed one of the first studies on multi-droplet impact in which they examined the bubble entrainment caused by the impact of the first 
two droplets created from a jet undergoing break up. They found that bubble entrainment is affected by droplet diameter, impact velocity, and timing between impacts. Hurd et al. (2015) were among the first to show that if the temporal frequency of the multi-droplet stream is high enough sequential droplets hitting the free surface in the same place create nested cavities, with each successive droplet forming a cavity at the base of the preceding cavity. They named this a matryoshka cavity after the Russian nesting dolls. We will also refer to the nested cavities created by multi-droplet impacts as matryoshka cavities. Bouwhuis et al. (2016) studied the same event for micrometer sized droplets impacting with frequencies in the range of $10-30 \mathrm{kHz}$ using boundary integral simulations with some experimental data for validation. In the regime studied, they showed that the expansion of the cavity is driven by inertia, but the collapse of the cavity is governed by surface tension (i.e., low Bo). They also showed that the downward cavity velocity approaches jet-like behavior as the spacing between the droplets decreases. Finally, they suggested that the maximum cavity radius and cavity depth at pinch-off scale with a modified $W e$. We show that their scaling only applies to one type of cavity seal (shallow seal) and does not generalize to our extensive experimental data set (Appendix A).

The existing body of work on rigid object impact has shown a variety of cavity shapes depending on the parameter space (Truscott et al., 2014). The depth of the cavity at pinch-off and the pinch-off time have been found to be functions of $W e$ and Bo (Duclaux et al., 2007; Aristoff \& Bush, 2009). Aristoff $\&$ Bush (2009) found four pinch-off types which all fall into distinct locations on a $B o-W e$ plot. At the lowest $W e$ they describe quasi-static seal, in which pinch-off occurs on or very near the sphere surface. As $W e$ increases shallow seal is seen, in which a much larger cavity forms that collapses near the pool surface under the influence of surface tension. Increasing $W e$ results in deep seal, where the cavity pinches-off approximately halfway between the surface and the sphere due to hydrostatic pressure. At the highest $W e$ a surface seal occurs, wherein the splash created upon impact collapses due to air pressure and surface tension (Marston et al., 2016) sealing off the cavity from further air entrainment and separating the cavity from the pool surface. Bouwhuis et al. (2016) revealed a shallow seal cavity type formed by successive micro-droplets similar to that formed by rigid sphere impact (Aristoff \& Bush, 2009). On the other hand, prior work on impacting liquid jets has observed only deep seal closure modes (Zhu et al., 2000, Oguz et al., 1992, 1995, Kersten et al., 2003, Soh et al., 2005: Qu et al., 2013). However, one might ask if impacting jets and multi-droplet streams can produce all of the cavity seal types observed for rigid sphere entry. Further, we suggest that other types of cavity seal exist that are unique to multi-droplet streams.

The aim of this experimental study is to investigate the dynamics of cavities created from multi-droplet streams and jets of water impacting on a deep pool. We explore multi-droplet and jet impacts over a much larger parameter space than has previously been investigated. The parameter ranges of multidroplets and jets (Table 1) span the dimensionless numbers: $B o \sim O\left(10^{-2}-10^{0}\right)$, $W e \sim O\left(10-10^{3}\right), F r \sim O\left(10^{2}-10^{4}\right), R e \sim O\left(10^{3}-10^{4}\right)$. These ranges suggest 
that for our experiments surface tension, gravitational, and inertial forces are important, but viscous effects are negligible. The experiments reveal that $W e$ and $B o$ scaling separate the cavity regimes and show that multi-droplet stream cavities are consistent with those formed by continuous jets. These cavity types encompass those seen previously for solid sphere entry, with three additional types for multi-droplet streams.

\section{Experimental setup and description}

Figure 2 a shows the experimental set up used for this study. A nozzle is placed $100-200 \mathrm{~mm}$ above a tank of water that is $730 \times 280 \times 350 \mathrm{~mm}^{3}$. Water flows to the nozzle through tubing that connects to a pressure reservoir that controls the velocity of the water exiting the nozzle. The nozzle forms a continuous stream, which is captured in a reservoir to prevent it from hitting the surface of the bath. We then remove the reservoir and allow the stream to fall continuously throughout the experiment. This method cannot control the length of the stream nor the final number of droplets. The diameter of the water jet $d_{j}$ (Fig. 2a) is varied by changing the nozzle diameter. The velocity of the jet exiting the nozzle $\left(U_{s}\right)$ is measured by imaging $50 \mu \mathrm{m}$ tracer particles that are mixed with the water; the average velocity across the jet profile is reported herein. The cavity velocity $\left(U_{c}\right)$ is measured by tracking the bottom of the cavity over time and fitting a line to the data; $U_{c}$ is taken as the slope of this line. In order to create multi-droplet streams with droplet diameter $d_{d}$, the nozzle is attached to a large shaker with adjustable frequency $f$ and amplitude to induce jet break-up as shown in Fig. 2a. The impact of the jet and droplet streams is viewed at $6000 \mathrm{fps}$ using two high speed cameras. One images below the surface to capture characteristics of the air cavity. The other views above the surface, from which diameters, velocities and frequencies of droplets are measured. These measurements reveal that the velocity of jets or droplets just prior to impact with the free-surface are equal for a given nozzle diameter and water flow rate; this impact velocity is referred to as $U_{s}$.

The achievable output parameters for multi-droplet streams (i.e., frequency, droplet diameter and velocity) are interrelated. Fig. $2 \mathrm{~b}$ and Table 1 show the range of combinations of $f, d_{d}$ and $U_{s}$ that can be produced for each nozzle diameter. The relationship of these variables can be derived from conservation of mass yielding,

$$
d_{d}=\left(\frac{3 d_{j}^{2} U_{s}}{2 f}\right)^{1 / 3} .
$$

This relationship is shown by the dashed line in Fig. $2 \mathrm{~b}$. The data follow this prediction very well except for the largest jet diameter $\left(d_{j}=1.49 \mathrm{~mm}\right)$, for which satellite droplets are formed but often land away from the stream impact location, thus reducing the average volume of the main droplets in the stream. Note, due to limitations of the setup, not all combinations of $d_{d}, f$ and $U_{s}$ can be achieved. 
a)

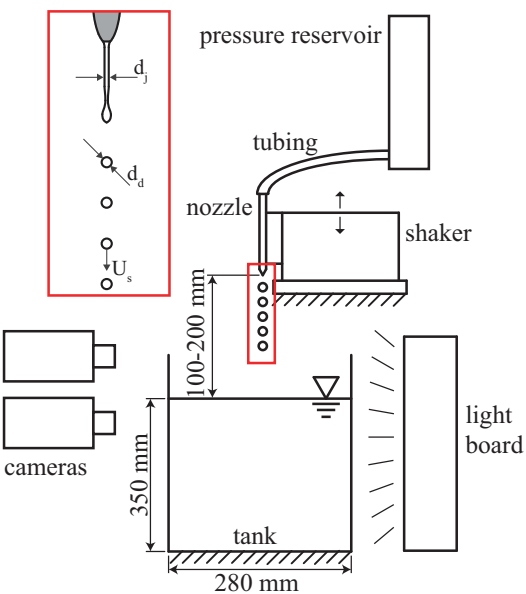

b)

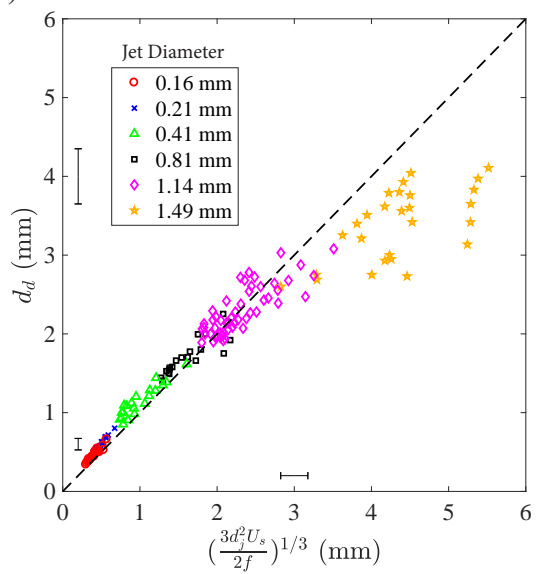

Figure 2: a) Schematic of the experimental setup with the inset indicating the mechanism for producing multi-droplet streams via jet breakup. The velocity of droplets $U_{s}$ has been observed experimentally to be largely constant over the fall height, and is equal to the jet velocity. b) The diameter of droplets $\left(d_{d}\right)$ in the multi-droplet streams is controlled by the nozzle diameter $\left(d_{j}\right)$, stream velocity $\left(U_{s}\right)$, and shaker frequency $(f)$ (Eq. ??). For a given nozzle diameter, the droplet diameter increases with decreasing $f$ and increasing $U_{s}$.

\begin{tabular}{|c|c|c|c|c|}
\hline $\begin{array}{l}\text { Jet Diameter } \\
d_{j}(\mathrm{~mm})\end{array}$ & $d_{d}(\mathrm{~mm})$ & $\begin{array}{l}\text { Droplets } \\
U_{s}(\mathrm{~m} / \mathrm{s})\end{array}$ & $f(\mathrm{~Hz})$ & $\begin{array}{c}\text { Jets } \\
U_{s}(\mathrm{~m} / \mathrm{s})\end{array}$ \\
\hline 0.16 & $0.34-0.66$ & $4.04-7.9$ & $1000-7000$ & - \\
\hline 0.21 & $0.45-0.80$ & $3.61-10.5$ & $1000-5000$ & - \\
\hline 0.41 & $0.85-1.62$ & $2.78-7.51$ & $300-4000$ & $4.24-7.83$ \\
\hline 0.48 & - & - & - & $3.12-6.51$ \\
\hline 0.71 & - & - & - & $3.94-9.69$ \\
\hline 0.81 & $1.40-2.25$ & $1.85-7.26$ & $200-2500$ & $2.91-5.32$ \\
\hline 1.14 & $1.89-3.08$ & $1.5-8.02$ & $80-2500$ & $3.69-8.01$ \\
\hline 1.49 & $2.60-4.11$ & $1.92-5.64$ & $100-500$ & $2.57-10.25$ \\
\hline
\end{tabular}

Table 1: Experimental parameter ranges for each jet diameter $d_{j}$ (Fig. 2 p). 
Figure 3 a shows an ideal matryoshka cavity created by a multi-droplet stream with relevant parameters given in Table 2 . The droplet spacing can be defined as $U_{s} / f$, where $f$ is the frequency of the droplet stream in the lab reference frame and is equivalent to the shaker frequency. The cavity geometry is defined by the depth from the undisturbed free-surface $h(t)$ and the cavity diameter $d_{c}(z, t)$. Additionally, there are three important cavity depths associated with relevant events. The first two can be seen in Fig. $4 \mathrm{k}$ at time $32.0 \mathrm{~ms}$ when pinch-off occurs. The depth at which cavity seal or pinch-off occurs, is denoted $h_{p}$. The depth of the bottom of the cavity at this time will be denoted as $h_{b}$. After all of the droplets from the stream have impacted and expanded the bottom of the cavity a new cavity depth is reached $h_{c}$ (Fig. 4 at $t=42.0 \mathrm{~ms}$ ).

For droplet streams impacting with low frequency, $f$, we will show that there is a dependent non-dimensional number that can help define the cavity regimes discussed more in $\S 3.1$. We coin this useful non-dimensional parameter the Matryoshka number and define it as

$$
M t=f_{a p} T_{\max }
$$

where $f_{a p}$ is the apparent frequency between droplet impacts and $T_{\max }$ is the time it takes for a cavity from a single droplet to reach maximum size. The apparent frequency experienced by the droplets impacting the bottom of the cavity is $f_{a p}=\left(1-U_{c} / U_{s}\right) f<f$, where $U_{c}$ and $U_{s}$ are depicted in Fig. 3 a. Measured values of $U_{c}$ and $U_{s}$ from image-based tracking methods are used to calculate $f_{a p}$ directly from experiments. When a single droplet impacts a pool of water it creates a cavity which expands radially outward from the point of impact. $T_{\max }$ occurs when the maximum cavity size is reached (Fig. 3p). Leng (2001) studied single droplet cavity formation and provided equations for estimating $T_{\max }\left(T_{\max }=0.524 \frac{d_{d}}{U_{s}} F r^{0.625}\right)$, which we use for all cases where $M t>10$. When $M t<10$ our own single droplet impact data was taken and the measured value of $T_{\max }$ used to calculate $M t$. More accurate values of $T_{\max }$ are required at low $M t$ since the measured vs. predicted values of $T_{\max }$ can vary by up to a factor of two or three. Given the equations for $T_{\max }$ and $f_{a p}$ above it is important to note that $M t$ is a dependent non-dimensional parameter as $f_{a p}$ and $T_{\max }$ are functions of $F r, \rho^{\prime}, \tilde{f}$ (defined below), $d_{d}, U_{s}$, and $f$. Nevertheless $M t$ is useful for classifying certain cavity types, especially cavities that occur when $M t<4$ as will be shown (§3.1).

To give more physical intuition to the significance of $M t$, consider the phenomena depicted in Fig. 3. The individual impacts of successive droplets divides the cavity into multiple sub-cavities. As the first droplet impacts the pool it creates a portion of the matryoshka cavity labeled sub-cavity 1 . The second droplet creates sub-cavity 2 , the third creates sub-cavity 3 and so on (Fig. 33). If droplets in a multi-droplet stream impact the bottom of the matryoshka cavity while the lowest sub-cavity is expanding then $M t>1$ (Fig. 3r). If droplets impact exactly at the time of momentum reversal of the lowest sub-cavity then $M t=1$, and if droplets impact after the momentum of the sub-cavity has already reversed then $M t<1$. The matryoshka number can be thought of as the 


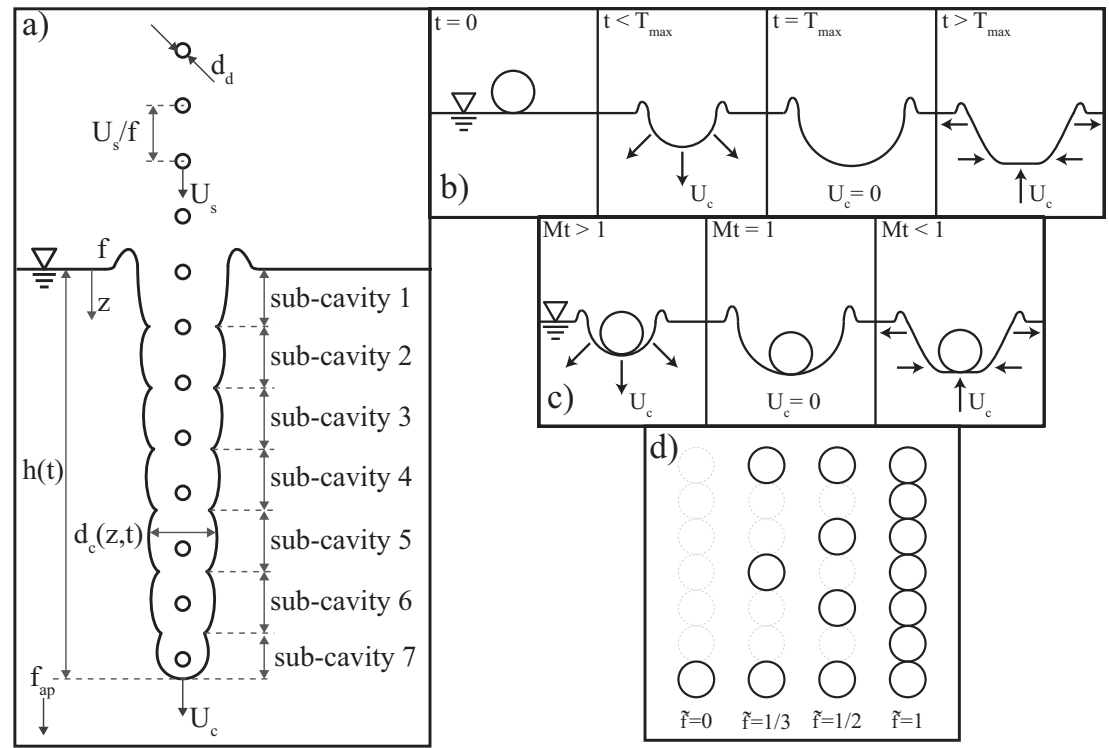

Figure 3: a) An idealized cavity created by a multi-droplet stream consists of several sub-cavities. b) A single droplet impact causes the cavity to expand at velocity $U_{c}$. The time of maximum cavity size $\left(T_{\max }\right)$ corresponds to $U_{c}=0$; for $t>T_{\max }$ the cavity is collapsing. c) The matryoshka number $(M t)$ is defined by the timing of the impact of the second droplet with respect to the maximum cavity size of the first. For example, for $M t=1$ the second droplet impacts the cavity at $T_{\max }$. d) The dimensionless frequency $\tilde{f}=f d_{d} / U_{s}$ defines the ratio of droplet diameter to spacing between droplets.

number of droplets that impact the bottom of the matryoshka cavity before the cavity from a single droplet impact has reached its maximum size.

A second important dimensionless frequency, denoted $\tilde{f}$ by Bouwhuis et al. (2016), is a ratio of droplet diameter to the center to center spacing between droplets, $\tilde{f}=f d_{d} / U_{s}$, taking the same form as the Strouhal number. As explained by Bouwhuis et al. (2016) and shown in Fig. 33, if $\tilde{f}=1$ droplets touch end to end, and the stream is reminiscent of a jet. If $\tilde{f}=1 / 3$, a third of the droplets remain in the stream. As $\tilde{f}$ approaches zero the droplets in the stream become separated by such a large distance that by the time the second droplet impacts the cavity has already become a flat surface again.

\subsection{Uncertainty}

Uncertainty in all image based measurements are calculated and the uncertainty bands in the figures represent the $95 \%$ confidence interval of the measurement (Coleman \& Steele, 2009). The uncertainty in calculated variables was often found to increase linearly with the variable. Where applicable, two bands are 


$\begin{array}{llll}\text { Variable } & \text { Description } & \text { Subscript } & \text { Description } \\ d & \text { diameter } & \{\}_{a p} & \text { apperent } \\ f & \text { frequency } & \{\}_{b} & \text { bottom of cavity at pinch-off } \\ h & \text { depth } & \{\}_{c} & \text { cavity } \\ t & \text { time } & \{\}_{d} & \text { droplet } \\ U & \text { velocity } & \{\}_{j} & \text { jet } \\ L, l & \text { stream length } & \{\}_{p} & \text { pinch-off } \\ & & \{\}_{s} & \text { stream (droplet or jet) }\end{array}$

Table 2: List of variables and their description.

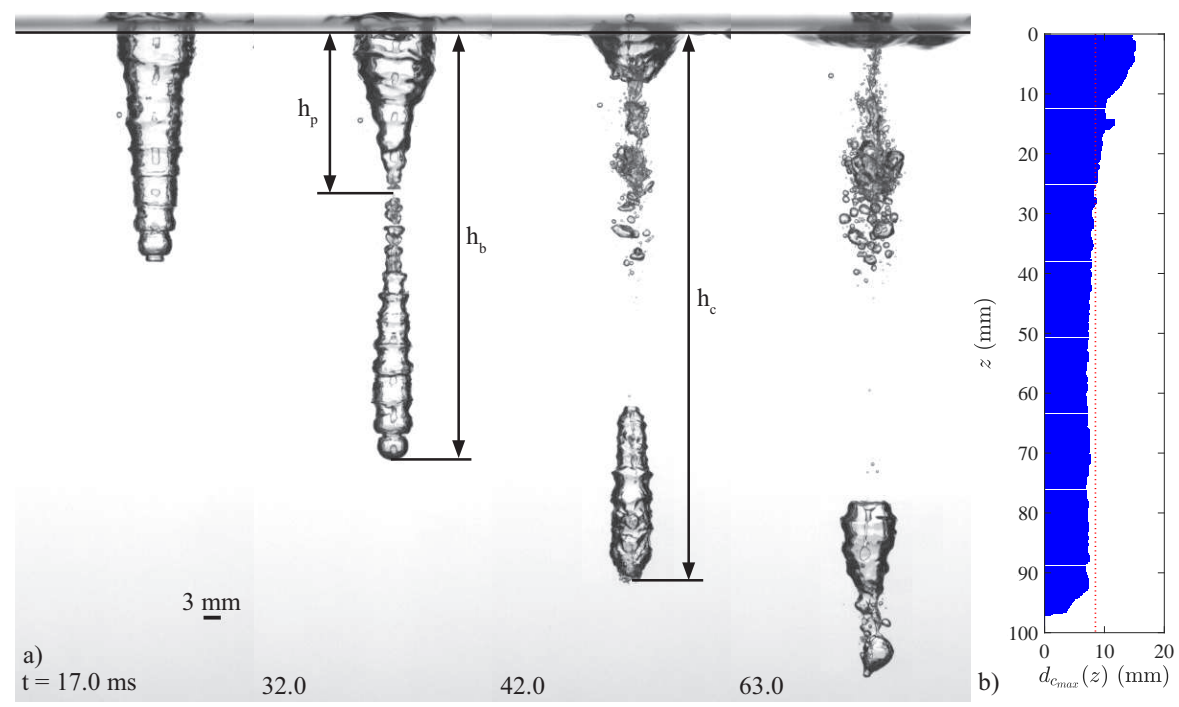

Figure 4: a) Four images from the impact of a multi-droplet water stream onto a pool of water. The pinch-off frame is shown at $t=32 \mathrm{~ms}$ with $h_{p}$ indicating the depth of the pinch-off point and $h_{b}$ indicating the depth of the cavity at this time. At $t=42 \mathrm{~ms}$ all of the droplets from the multi-droplet stream have impacted the cavity base creating a depth of $h_{c}$. At $t=63 \mathrm{~ms}$ all of the droplets from the downward jet created at pinch-off have impacted the cavity. b) The maximum cavity diameter over time is measured at discrete depths, yielding $d_{c_{\max }}(z)$. The average of these max diameters over all depths defines $d_{c}$ (red dotted line). 
placed on the extremes of the axes of a figure to show that the error is linearly increasing (e.g., Fig. 5), when only one band is present the mean uncertainty is shown.

\section{Cavity formation}

The basic sequence of events involved in a multi-droplet stream impact can be seen in Fig. 4a and supplemental video 1. As the first droplet hits the free surface a hemispherical cavity is formed. The next droplet impacts the base of the first cavity causing it to expand downward. This process continues until the cavity pinches together on the droplet stream sealing it off from more incoming droplets. Even after this pinch-off event occurs droplets in the lower portion of the cavity continue impacting the bottom, pushing it deeper into the pool while maintaining roughly the same volume since pinch-off. Once all of the droplet stream has impacted, droplets formed from the downward jet created at pinch-off will continue to impact the cavity bottom, pushing it even deeper into the pool. These droplets created from the downward jet are usually much less consistent in diameter and trajectory and thus cause the cavity to evolve in a much more chaotic manner. Our experimental observations conclude that the downward jet does not affect the droplet stream as it forms after the stream has passed the pinch-off point and has a velocity approximately less than or equal to the stream velocity (e.g., supplemental videos 1 and 7 ). This sequence of events is similar for the impact of a jet (supplemental video 2).

Figure 5 shows that the ratio of the cavity diameter to jet or droplet diameter increases linearly with impact velocity. Oguz et al. (1995) and Zhu et al. (2000) claim that $d_{c} / d_{j}$ is constant around 2.41 for jets, irrespective of impact velocity. This finding is inconsistent with the data presented here, but could be explained by the small velocity ranges studied in those works, $0.88-2.64 \mathrm{~m} / \mathrm{s}$ and $0.96-1.87$ $\mathrm{m} / \mathrm{s}$ respectively.

The cavity diameter produced by jets is always larger than the cavity diameter formed by multi-droplet streams at the same velocity (Fig. 5p. We find a common factor of approximately 1.75 by taking the ratio of the least squares regressions of the jet data to the multi-droplet data. Because the cavity diameter affects other cavity dynamics, such as the relative magnitude of the gravitational, inertial, and surface tension forces, we will include this scaling factor by defining a modified droplet diameter of $d_{d}^{*}=d_{d} / 1.75$. The inset of Fig. 5 shows that this re-scaling collapses the multi-droplet data onto the curve for jets, where $d_{s}=d_{d}^{*}$ for droplets and $d_{s}=d_{j}$ for jets. Based on this empirical finding, we re-define the Bond, Weber, and Froude numbers of the multi-droplet streams as $B o^{*}=\rho g d_{s}^{2} / \sigma, W e^{*}=\rho U_{d}^{2} d_{s} / \sigma$, and $F r^{*}=U_{d}^{2} / g d_{s}$, respectively. For the remainder of the paper, we may use the ${ }^{*}$ symbol for jets and droplets for ease of discussion, but when used for jets the $d_{j}$ is not modified. While we are currently unable to offer an analytic explanation for the factor of 1.75 , the empirical evidence shows self-consistency within our wide ranged experimental data set. 


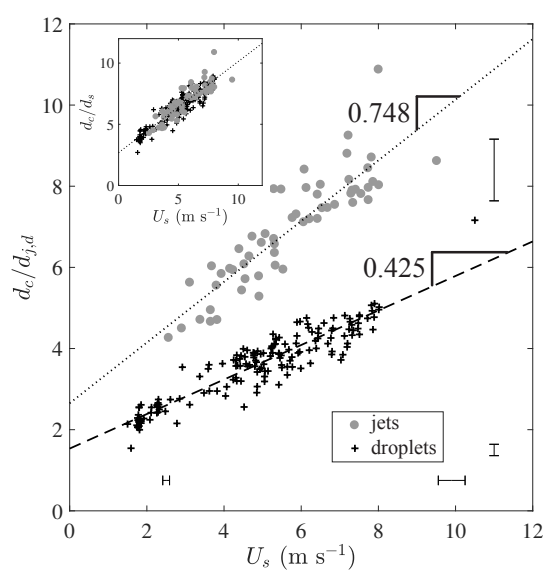

Figure 5: Normalizing cavity diameter $\left(d_{c}\right)$ by jet diameter $\left(d_{j}\right)$ collapses the jet data onto a line that is a function of impact velocity $\left(U_{s}\right)$. A line with different slope and intercept is found for multi-droplet cases. We define a modified droplet diameter $d_{d}^{*}=d_{d} / 1.75$, which collapses the normalized cavity diameter data onto a single line for multi-droplet and jet streams (inset).

Given previous work on the water impact of spheres (Aristoff \& Bush, 2009), one might expect different classes of cavities to form for multi-droplet impact, depending on the parameter regime. Indeed, Bouwhuis et al. (2016) observed cavities formed by micro-droplet streams that are best described as shallow seal. Here we observe six distinct cavity types, three occurring for multi-droplet streams at low matryoshka number and three more that occur for both droplet streams and jets (Fig. 63). The first three occur only when droplets impact close to the maximum cavity size of the preceding sub-cavity (i.e., when $M t \sim O(1)$ ). In these cases, sub-cavities maintain some independence from the matryoshka cavity as a whole and their independent behavior determines when and where cavity seal will occur. Thus, we call them sub-cavity seals. The other three occur for both droplet streams and jets and are the same type of cavity seals or pinch-offs observed by Aristoff \& Bush (2009) for hydrophobic spheres at low Bond number. We will discuss each of these in turn.

\subsection{Sub-cavity collapse}

As shown in Fig. 3 3 , as a single droplet impacts the water surface it creates a cavity that expands downward and radially outward from the point of impact (Engel, 1966). After reaching its maximum size the bottom of the cavity collapses radially inwards and upwards while the top widens under the flattening influence of surface tension. When $M t<1$, the momentum of the first sub-cavity has already reversed and is in the upward direction by the time the second droplet impacts its base (Fig. $7 \mathrm{~h}, t=14.7 \mathrm{~ms}$ ). Upon impact the sec- 


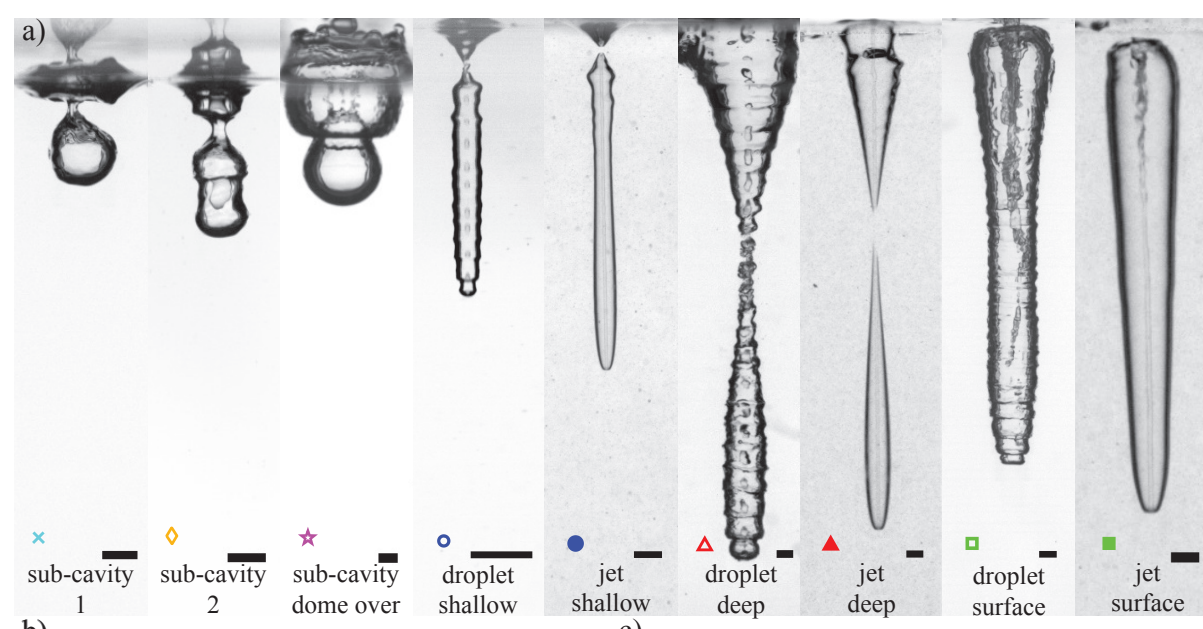

b)
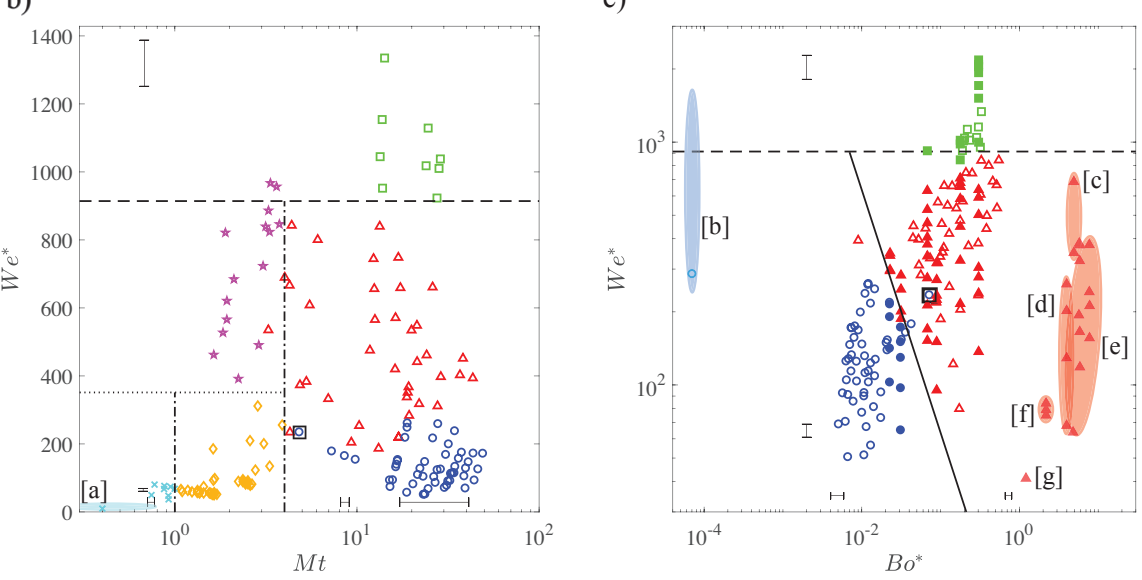

Figure 6: a) Multi-droplet streams produce six cavity seal types, three of which - shallow seal, deep seal and surface seal - are common to jet streams and rigid sphere impact. Scale bars at the bottom of each image are $3 \mathrm{~mm}$. b) The six cavity seal types for multi-droplet streams can be separated by a $M t-W e^{*}$ regime diagram. The horizontal dashed line represents the lowest $W e$ found by Franz (1959) for which dome over occurs for a single droplet (line also shown in c; note that $W e$ from Franz $(1959)$ has been modified using $\left.d_{d}^{*}\right)$. Sub-cavity 1 collapse only occurs for $M t<1$, while sub-cavity 2 collapse only occurs between $1<M t<4$. Sub-cavity dome over occurs for $W e^{*} \gtrsim 350$ and $M t<4$; the horizontal dotted line is to guide the eye. c) Shallow seal, deep seal and surface seal are better sorted on a $B o^{*}-W e^{*}$ plot. The solid line in c) is found by equating the dimensionless pinch-off times for deep and shallow seal (Eq. ??). Using the modified diameter $d_{d}^{*}$ causes the cavity seal regime boundaries to align for multi-droplet and jet streams. Data from previous studies are plotted and match the expected cavity seal regimes; [a] Bick et al. (2010), [b] Bouwhuis et al. (2016), [c] Qu et al. (2013), [d] Zhu et al. (2000), [e] Soh et al. (2005), [f] Kersten et al. (2003), and [g] Oguz et al. (1992). Symbols and data are the same for all figures throughout the paper. 


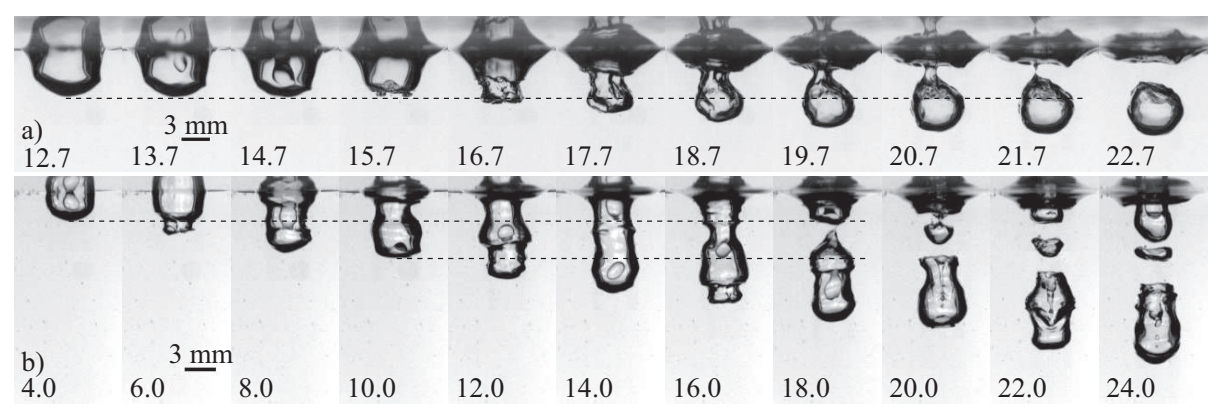

Figure 7: Two types of sub-cavity collapse: sub-cavity 1 collapse a), and subcavity 2 collapse b). a) For $M t<1$, the second droplet impacts the cavity at $t=14.7 \mathrm{~ms}$ after sub-cavity 1 has begun to collapse $\left(f=80 \mathrm{~Hz}, U_{s}=1.77 \mathrm{~m}\right.$ $\mathrm{s}^{-1}, d_{d}=3.08 \mathrm{~mm}, W e^{*}=77, B o^{*}=0.4, M t=0.88$, and $\left.\tilde{f}=0.14\right)$. The dashed line indicates the location of the bottom of sub-cavity 1 at its maximum depth. b) For $1<M t<4$, the second droplet impacts the first sub-cavity at $t=4.0 \mathrm{~ms}$, the third droplet impacts at $t=10.0 \mathrm{~ms}$ and so on. The overall cavity is still expanding when each successive droplet impacts the cavity, thus, enabling the cavity to grow larger in the vertical direction $(f=300 \mathrm{~Hz}$, $U_{s}=1.80 \mathrm{~m} \mathrm{~s}^{-1}, d_{d}=2.18 \mathrm{~mm}, W e^{*}=56, B o^{*}=0.21, M t=1.55$, and $\tilde{f}=0.36$ ). The cavity collapse occurs just below the bottom of sub-cavity 1 but above the bottom of sub-cavity 2. Dashed lines indicate the bottoms of subcavities 1 and 2 just prior to impact by the subsequent droplet. Numbers at the bottom of each frame indicate the time after initial impact in milliseconds. Corresponding positions in the regime diagrams can be seen in Fig 6b.Videos for a) and b) are shown in supplemental videos $3 \& 4$ respectively. 
ond droplet spreads and transfers downward momentum to the cavity but only over the small area that it impacts. The rest of the first sub-cavity is not impacted by the second droplet and continues its upward collapse while the second sub-cavity expands downward (Fig. $7 \mathrm{p}, t=16.7$ - $20.7 \mathrm{~ms}$ ). These opposing velocities cause sub-cavities 1 and 2 to separate from each other (Fig. $7 \mathrm{a}, t=21.7$ $\mathrm{ms}$ ). In this case cavity seal always occurs between the undisturbed free surface and the bottom of sub-cavity 1 and hence we will call this type of cavity seal sub-cavity 1 collapse.

If we maintain $W e^{*} \lesssim 350$ and increase the matryoshka number, $1<M t<4$, each successive droplet impacts while the preceding sub-cavity is still expanding downward (Fig. $7 \mathrm{~b}, t=4 \mathrm{~ms}$ ). This gives the droplet more time to spread over the base of the sub-cavity, transferring the momentum to a larger area and counteracting the impending momentum reversal in the vertical direction, in contrast to $M t<1$. Although this transfers downward momentum it does not counteract the impending momentum reversal in the radial direction. The pinchoff location is determined by which sub-cavity is first to collapse (1 or 2$)$. The difference between sub-cavities 1 and 2 is in the boundary conditions. The top of sub-cavity 1 resides on the free-surface which allows it to widen for a longer time. The widening of the top of sub-cavity 1 combined with the expansion of the bottom due to the impact of the second droplet delays the collapse of subcavity 1 . On the other hand, the top of sub-cavity 2 is driven radially inward by surface tension and hydrostatic pressure, resulting in a collapse time that is sufficiently small to pinch-off before sub-cavity 1 (Fig. $7 \mathrm{p}, t=18 \mathrm{~ms}$ ). In this regime $\left(1<M t<4, W e^{*} \lesssim 350\right)$ pinch-off always occurs between the bottoms of sub-cavity 1 and 2 . Hence, we call this pinch-off type sub-cavity 2 collapse.

Increasing $W e^{*}$ above 350 while maintaining $M t<4$, we see in Fig. 8 that a splash crown forms at the bottom of each sub-cavity upon impact by the subsequent droplet. When conditions are right, the splash crown of each subcavity will collapse inward on itself and dome over, sealing off air flow between sub-cavities. We call this event sub-cavity dome over. In Fig. 8 8 we examine a cavity with $M t=1.84$ and $W e^{*}=527$. At $t=31 \mathrm{~ms}$ the splash crown at the base of the second sub-cavity is about to dome over. At $33.5 \mathrm{~ms}$ a droplet has impacted this dome over event morphing into a downward moving splash. Meanwhile the bottom of sub-cavity 2 continues its upward motion and the cavity collapses inward near the dome over event eventually leading to a full pinch-off that detains further droplets from entering into the lower portion of the cavity $(t=36-41 \mathrm{~ms})$.

We see different behavior looking at a case with a slightly higher $M t$, but still below four. In Fig. 8 8 at $33.2 \mathrm{~ms}$ the splash crown at the base of the third sub-cavity domes over. As the next droplet continues downward, it breaks through this domed-over splash crown and morphs into a downward moving splash $(\mathrm{t}=34.8 \mathrm{~ms})$, which then impacts the dome over event at the base of the next sub-cavity, sub-cavity $4(t=34.8-36.5 \mathrm{~ms})$. Because $M t$ is higher than the previous case, the sub-cavities have insufficient time to collapse. Hence, incoming droplets continue to break through each sub-cavity dome over event and the cavity eventually pinches off with a deep seal, as discussed below in 


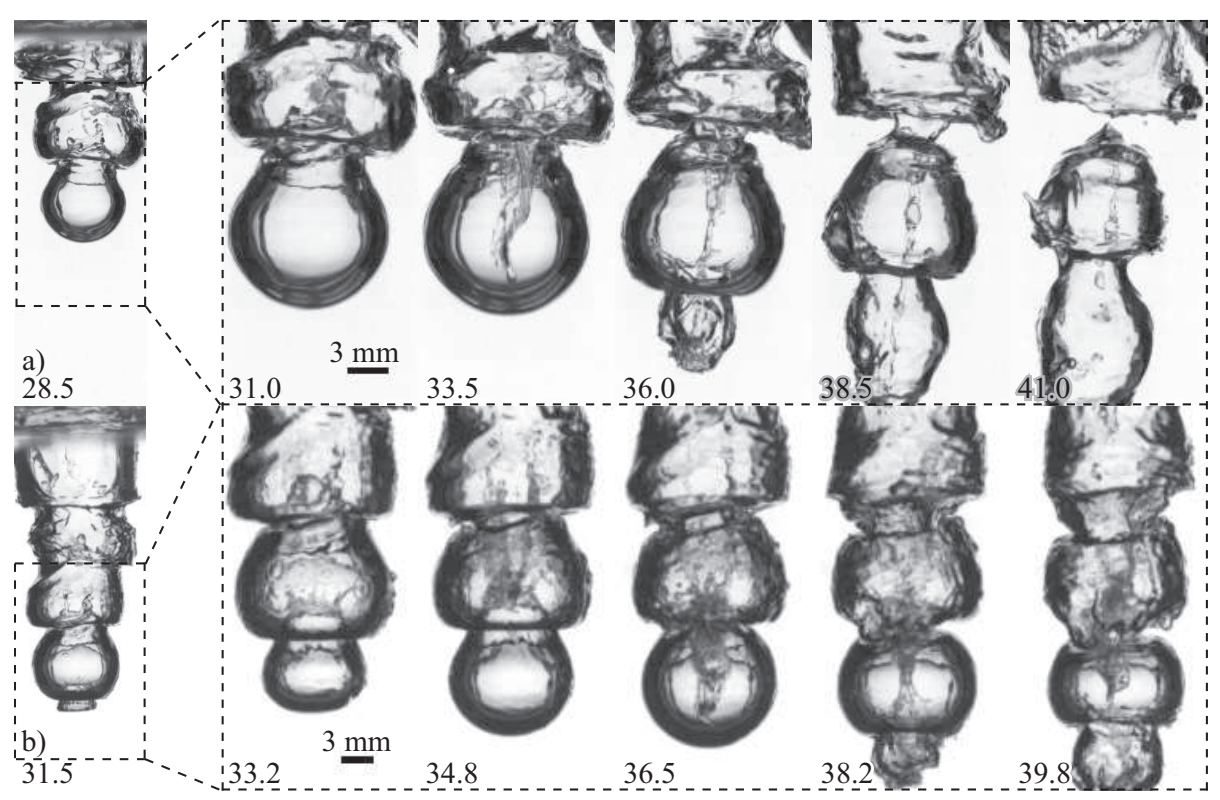

Figure 8: Two cases of sub-cavity dome over. When $M t<4$ and $W e^{*} \gtrsim 350$ splash crowns form at the base of each sub-cavity and sometimes dome over. a) When $M t$ is at the lower end of this range the cavity fully pinches off at the dome over positions detaining further droplets from entering into the lower portion of the cavity $\left(f=100 \mathrm{~Hz}, U_{s}=4.41 \mathrm{~m} / \mathrm{s}, d_{d}=3.42 \mathrm{~mm}, W e^{*}=527, B o^{*}=0.52\right.$, $M t=1.84$, and $\tilde{f}=0.08)$. b) As $M t \rightarrow 4$ droplets break through the subcavity dome overs reopening the cavity and preventing a full pinch-off $(f=200$ $\mathrm{Hz}, U_{s}=5.45 \mathrm{~m} / \mathrm{s}, d_{d}=3.76 \mathrm{~mm}, W e^{*}=886, B o^{*}=0.63, M t=3.27$, and $\tilde{f}=0.14)$. Numbers at the bottom of each frame indicate the time after impact in milliseconds. The first image in each sequence shows a zoomed out view of the cavity and each following image shows a zoomed in view of the cavity of the area indicated by the dashed box. Corresponding positions in the regime diagrams can be seen in Fig 6 b. Videos for a) and b) are shown in supplemental videos $5 \& 6$ respectively. 
$\S 3.2$, and seen in supplemental video 6 . The division between these three subcavity regimes can be seen on the $M t$ verse $W e^{*}$ plot shown in Fig. 6b. Note that for $M t<4$ and $W e^{*}>914$, it is unclear if the sub-cavity dome over regime persists; however, based on the work of Franz (1959) we suspect that sub-cavity 1 would begin to dome over in this region effectively producing a surface seal.

\subsection{Sphere-like cavity collapse}

When $M t>4$, the cavity velocity becomes more stable $(\S 4)$ and the matryoshka cavity assumes a more continuous form as droplets impact in rapid succession on newly formed sub-cavities. In this regime the three different types of cavity seals, seen for both multi-droplet streams and jets, are best shown on a $B o^{*}$ $W e^{*}$ plot in Fig. 6. These three cavity seal types are also seen in the water entry of hydrophobic spheres (Aristoff \& Bush, 2009) and thus we call them sphere-like cavity seals.

The first of the sphere-like cavities occurs at the lowest $W e^{*}$ and $B o^{*}$ and is called shallow seal. Fig. 9 shows an image sequence of this event for a multidroplet stream and a jet. As the droplet stream or jet impacts, a long, narrow, cylindrical cavity forms. A capillary wave forms near the surface, which travels down the cavity walls eventually causing it to pinch together at a depth on the order of the capillary length, as shown at $5.5 \mathrm{~ms}$ in Fig. 9a for a droplet stream and at $13.3 \mathrm{~ms}$ in Fig. $9 \mathrm{p}$ for a jet. This is the same sequence of events described by Aristoff \& Bush (2009) for shallow seal of cavities created by small spheres. This event is dominated by capillary forces as described by the small $B o^{*}$ for which it occurs. Although not specifically called shallow seal in their paper, this is the experimental regime that Bouwhuis et al. (2016) studied. Following the shallow seal event, the cavity may experience a deep seal pinch-off as shown in Fig. $9 \mathrm{p}$ at $16.3 \mathrm{~ms}$.

An interesting phenomenon that can occur for jets in the shallow seal regime is the break up of the jet after pinch-off due to disturbances that grow from the Rayleigh-Plateau instability (Lin \& Reitz, 1998). When the cavity walls pinch-off and impact the sides of a small diameter jet, the disturbance can be sufficiently large to cause the jet to break up into an uneven droplet stream more quickly than normal. This break up is seen in Fig. 9b at $14.8 \mathrm{~ms}$ and explains why the smooth cavity created by the jet becomes irregular after pinch-off.

As $W e^{*}$ and $B o^{*}$ increase, inertial and pressure forces become more important and cavities enter the deep seal regime. Unlike the shallow seal event, the cavity walls pinch together approximately halfway between the surface and the bottom of the cavity at the time of pinch-off (Fig. 10p. The predominate force driving this event is hydrostatic pressure as described by the higher $B o^{*}$. While the jet literature cited in the introduction does not specify the type of cavity seal, the images and range of $B o$ and $W e$ reported in these references indicate deep seal cavities (Zhu et al., 2000, Oguz et al., 1992; Kersten et al., 2003; Soh et al., 2005: Qu et al., 2013). As shown in Fig. 6e, these prior studies fall in the deep seal regime on the $B o^{*}-W e^{*}$ diagram.

The division between the shallow and deep seal regimes shown by the solid 


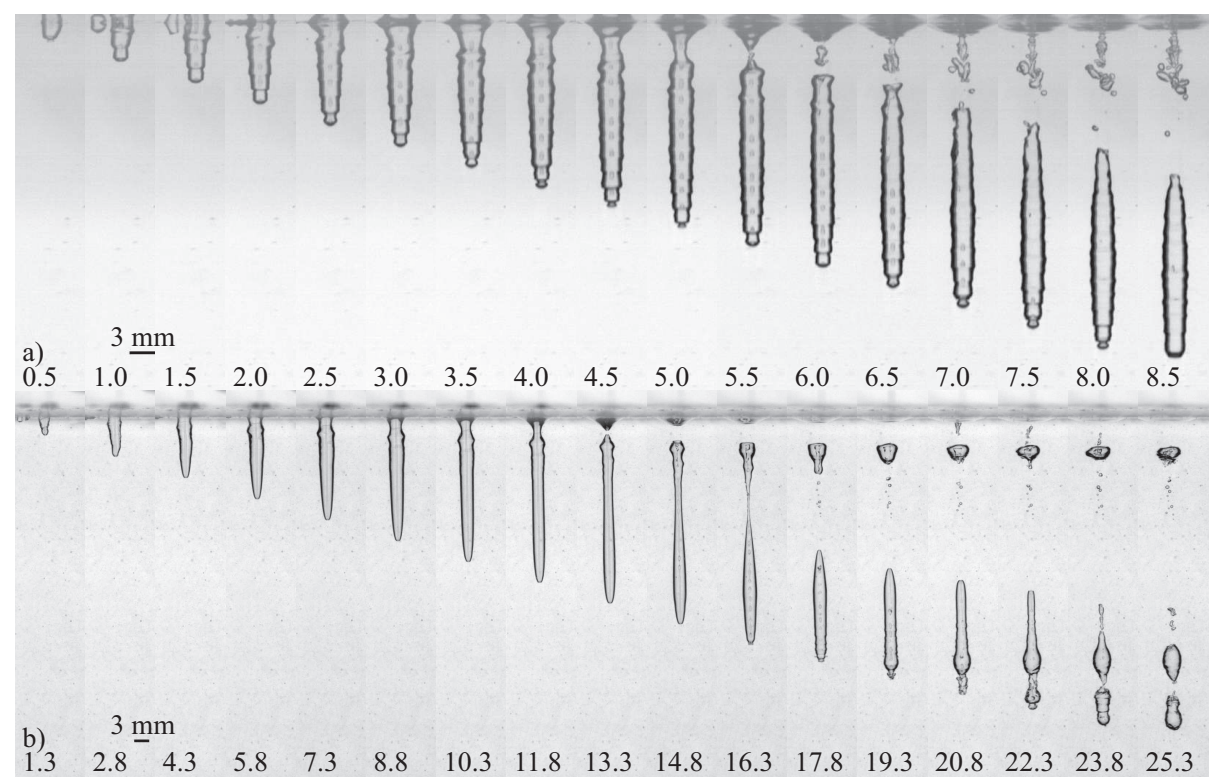

Figure 9: Shallow seal shown for two stream types. A capillary wave forms near the surface and travels downward until pinch-off occurs at a depth on the order of the capillary length. a) A cavity created by a multi-droplet stream pinches off with a shallow seal for small $B o^{*}\left(f=6000 \mathrm{~Hz}, U_{s}=6.39 \mathrm{~m} \mathrm{~s}^{-1}\right.$, $d_{d}=0.39 \mathrm{~mm}, W e^{*}=128, B o^{*}=0.0069, M t=39.7$, and $\left.\tilde{f}=0.37\right)$. b) A cavity created by a jet experiences first shallow and then deep seal $\left(U_{s}=5.78\right.$ $\mathrm{m} \mathrm{s}^{-1}, d_{j}=0.41 \mathrm{~mm}, W e^{*}=190$, and $\left.B o^{*}=0.023\right)$. After shallow seal pinchoff, the jet is perturbed and begins to break up into a droplet stream from the Rayleigh-Plateau instability $(t \geq 14.8 \mathrm{~ms}$ ). Numbers at the bottom of each frame indicate the time after impact in milliseconds. Corresponding positions in the regime diagrams can be seen in Fig $6 \mathrm{~b} \&$ c. Videos for a) and b) are shown in supplemental videos $7 \& 8$ respectively. 


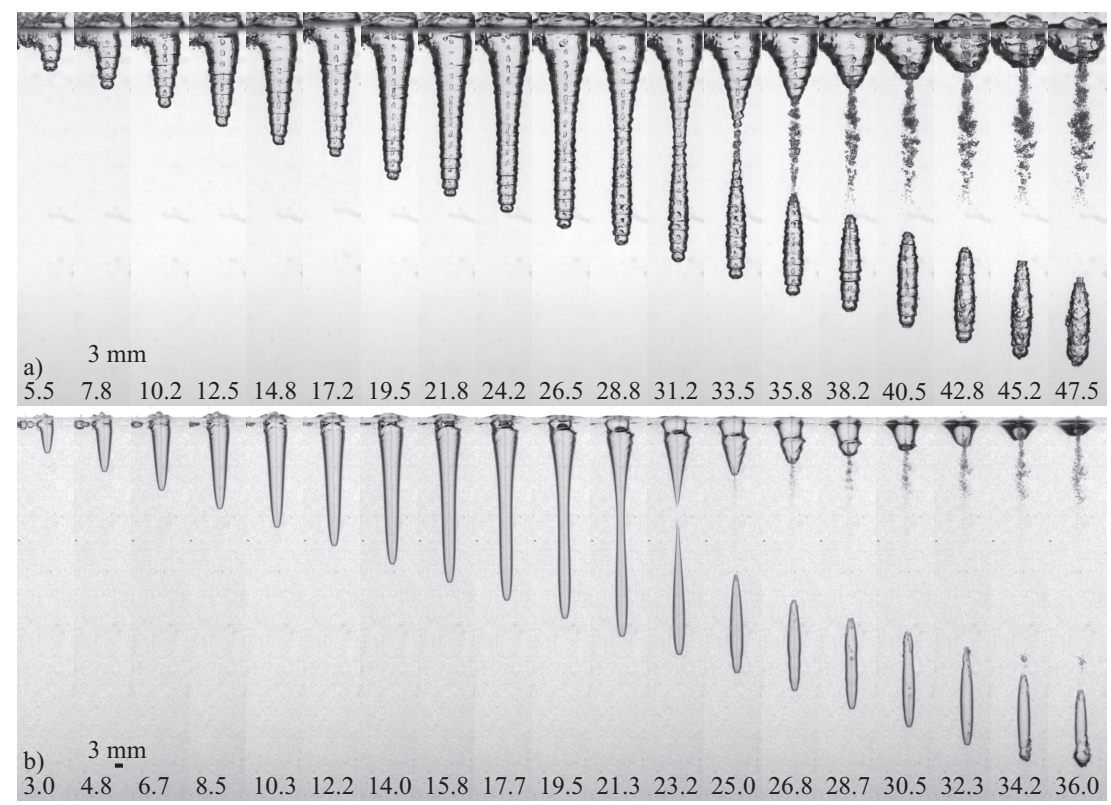

Figure 10: Deep seal shown for two stream types. Pinch-off occurs approximately halfway between the free-surface and cavity bottom. a) A cavity formed by a multi-droplet stream with higher $B o^{*}$ and $W e^{*}$ pinches off in a deep seal. $\left(f=1500 \mathrm{~Hz}, U_{s}=6.59 \mathrm{~m} / \mathrm{s}, d_{d}=2.17 \mathrm{~mm}, W e^{*}=748, B o^{*}=0.21\right.$, $M t=16.9$, and $\tilde{f}=0.49)$. b) A cavity formed by a jet at a moderate $B o^{*}$ pinches off in a deep seal $\left(U_{s}=7.09 \mathrm{~m} / \mathrm{s}, d_{j}=0.71 \mathrm{~mm}, W e^{*}=527\right.$, and $\left.B o^{*}=0.068\right)$. Because $B o^{*}$ is relatively small for this jet impact, we still observe the downward moving capillary wave near the surface seen for shallow seal. Numbers at the bottom of each frame indicate the time after impact in milliseconds. Corresponding positions in the regime diagrams can be seen in Fig 6 b \& c. Videos for a) and b) are shown in supplemental videos 1 \& 2 respectively. 
a)

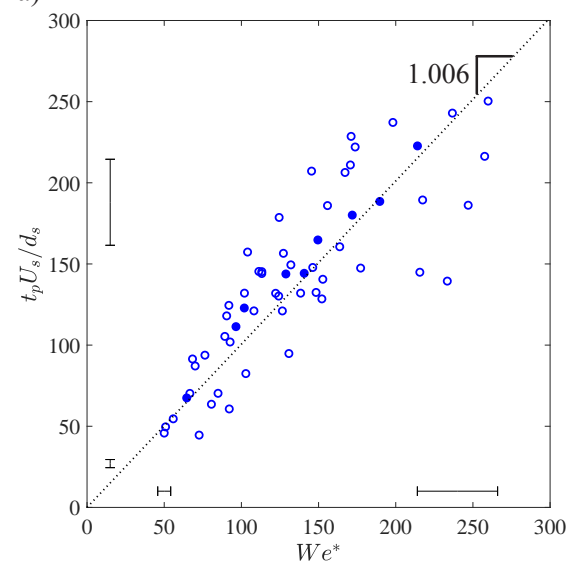

b)

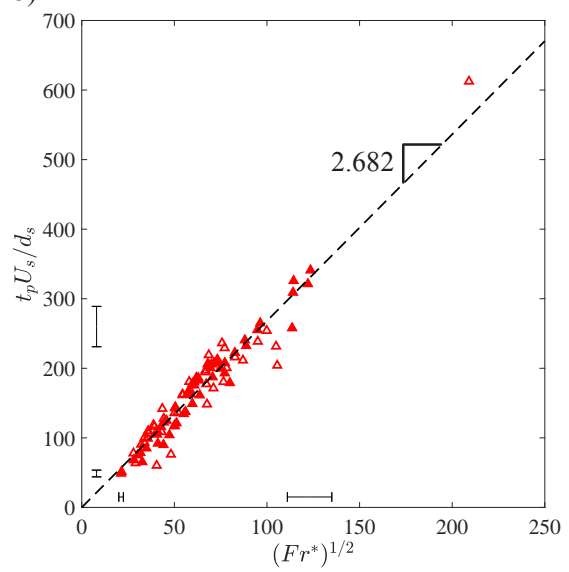

Figure 11: Dimensionless pinch-off time for a) shallow seal and b) deep seal. The slopes of the respective fits are used in Eq. ?? to compute the boundary between shallow and deep seal shown on Fig. 6r. Symbols are outlined in the legend of Fig. 6a.

line in Fig. 6re are found by equating the dimensionless pinch-off times for shallow and deep seal (Aristoff \& Bush, 2009). Pinch-off time is non-dimensionalized as $t_{p} U_{s} / d_{s}$ in Fig. 11. Shallow seal is driven by surface tension, thus the dimensionless pinch-off time can be predicted using $W e^{*}$ (Fig. 11a), whereas deep seal is driven by hydrostatic pressure and thus pinch-off time scales with $\mathrm{Fr}^{*}$ (Fig. 11b). Equating the shallow and deep non-dimensional pinch-off times gives the equation for the solid black line in Fig. 6. separating the shallow and deep seal regimes as

$$
W e^{*}=\frac{m_{\text {deep }}^{2}}{m_{\text {shal }}^{2}}\left(B o^{*}\right)^{-1},
$$

with $m_{\text {shal }}$ equal to the slope of the pinch-off time fit for shallow seal and $m_{\text {deep }}$ equal to the slope of the fit for deep seal, as shown in Fig. 11. It is worth noting that modifying the droplet diameter as discussed in $\S 3$ causes the dimensionless pinch-off times to fall on a common line for both jets and droplet streams. This again indicates that the cavity diameter governs the pinch-off characteristics.

An interesting phenomenon can occur for multi-droplet impacts that border on three cavity seal regimes. For example, on Fig. 6c, the blue circle inside the black square corresponds to a shallow seal event with $B o^{*}=0.073$ and $W e^{*}=$ 234 , yet the location of the symbol falls in the deep seal regime. Furthermore, for this case $M t=4.84$, which places the case close to the sub-cavity 2 collapse regime. Because the pinch-off for shallow seal and sub-cavity 2 collapse occurs at approximately the same depth below the surface, we suggest that the two mechanisms associated with these two types of cavity seal have an additive effect resulting in a shallow pinch-off. In this case shallow seal was closely followed by 
deep seal, reflecting the intersection of three regimes of cavity seal for this case.

As $W e^{*}$ increases above that seen for deep seal, the low air pressure created by the velocity of the stream and surface tension forces cause the splash crown to collapse inward on itself, or dome over, sealing the cavity off from further air entrainment (Marston et al., 2016). This regime is called surface seal. The cavity then pulls away from the free surface entering deeper into the pool. Deep seal often follows surface seal splitting the cavity in two. This sequence of events can be seen in Fig. 12 with dome over occurring at $12.0 \mathrm{~ms}$ in a) for the multidroplet stream and at $8.3 \mathrm{~ms}$ in b) for the jet. Even after the dome over has occurred the stream of water continues to penetrate into the cavity, but has now been distorted by passing through the splash crown. The cut off between the deep seal and surface seal regimes occurs at $W e^{*}=914$, which is the same $W e^{*}$ (following modification of the droplet diameter) at which Franz (1959) saw dome over beginning to occur for single droplet impacts. This implies that the dome over event is predominately caused by the impact of the first droplet and thus can be thought of as either a sub-cavity 1 dome over or a surface seal event from a sphere-like water entry.

\section{Cavity velocity}

We will now examine the downward growth rate of the cavity. In Fig. 13 a the unsteady growth of four different cavities in time is presented. The effect of each individual droplet impact can be seen in the cavity depth evolution, with the downward velocity varying over the period of expansion of each sub-cavity, $T_{\max }$. Thus, in describing the downward velocity of the cavity we can discuss two parts: the average cavity velocity (captured by linear fits) and the oscillating cavity velocity.

Based on the work of Birkhoff \& Zarantonello (1957) (pg. 16) we can develop a model that predicts the cavity velocity, $U_{c}$. To begin we neglect gravity during impact and consider the impact of a jet in a reference frame moving at the velocity of the cavity bottom. Noting that the pressure of the two media (jet and pool) at the stagnation point are balanced and applying the Bernoulli equation along the axial stream lines in both media, the unknown pressure at the stagnation point can be eliminated. This approach yields

$$
\frac{1}{2} \rho\left(U_{s}-U_{c}\right)^{2}=\frac{1}{2} \rho U_{c}^{2},
$$

and rearranging gives

$$
\frac{U_{c}}{U_{s}}=\frac{1}{2} .
$$

Bouwhuis et al. (2016) used computation and experimental methods to show that for multi-droplet streams $U_{c} / U_{s}$ approaches $1 / 2$ as $\tilde{f}$ approaches unity. We can now modify the energy balance in Eq. ?? accounting for the intermittent 


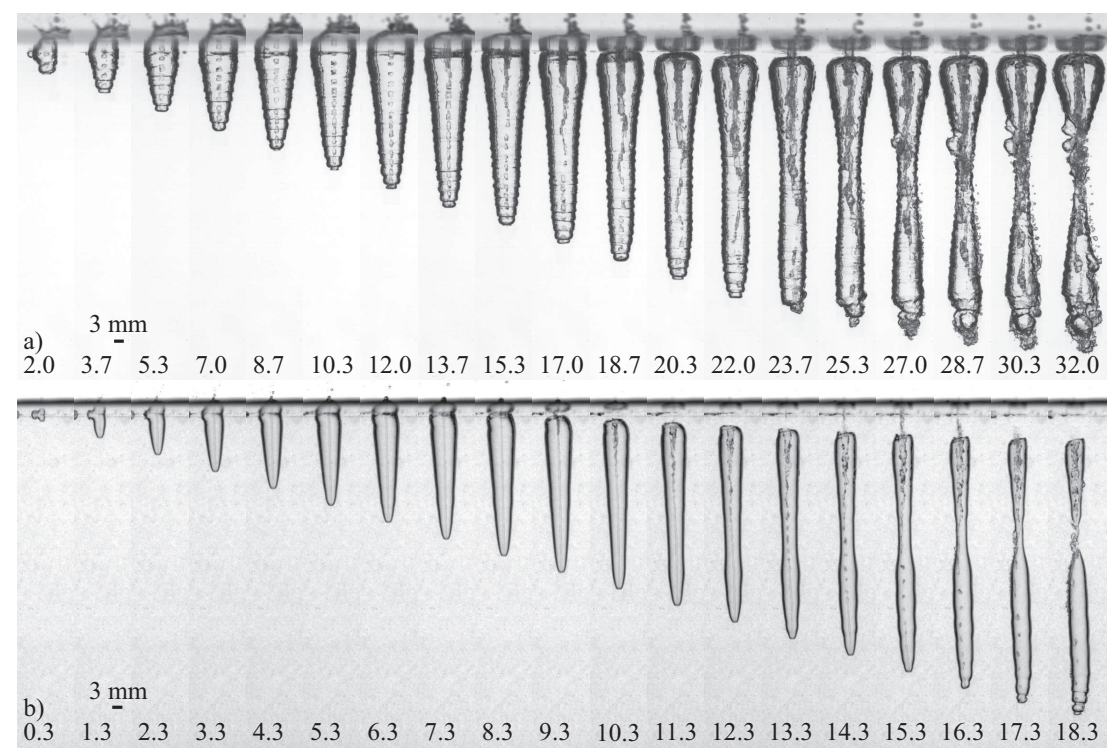

Figure 12: Surface seal for two types of stream impacts. a) The splash crown of a cavity created by a multi-droplet stream domes over at $t=12.0 \mathrm{~ms}$ resulting in surface seal $\left(f=2500 \mathrm{~Hz}, U_{s}=7.51 \mathrm{~m} \mathrm{~s}^{-1}, d_{d}=2.06 \mathrm{~mm}, W e^{*}=923\right.$, $B o^{*}=0.19, M t=28$, and $\left.\tilde{f}=0.69\right)$. b) The same phenomenon is observed for a cavity created by a jet with dome over occurring at $t=8.3 \mathrm{~ms}\left(U_{s}=9.69 \mathrm{~m}\right.$ $\mathrm{s}^{-1}$ and $d_{j}=0.71 \mathrm{~mm}, W e^{*}=921$, and $\left.B o^{*}=0.068\right)$. Numbers at the bottom of each frame indicate the time after impact in milliseconds. Corresponding positions in the regime diagrams can be seen in Fig $6 \mathrm{~b} \& \mathrm{c}$. Videos for a) and b) are shown in supplemental videos $9 \& 10$ respectively. 


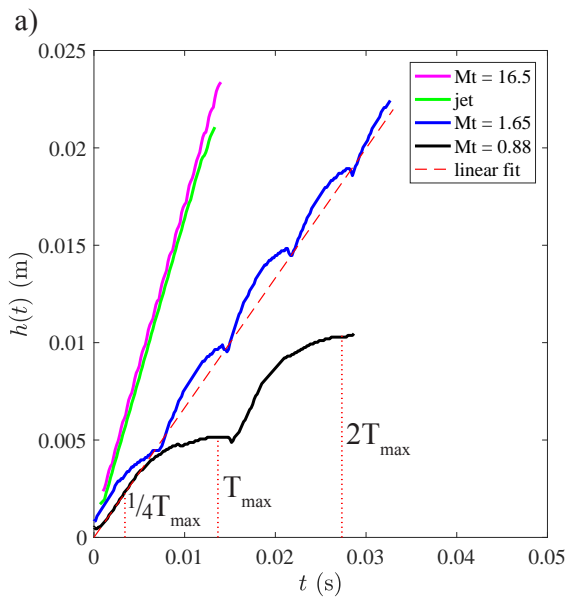

b)

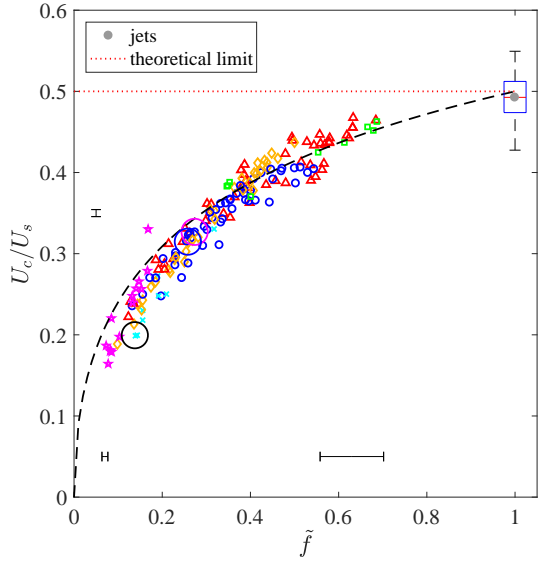

c)

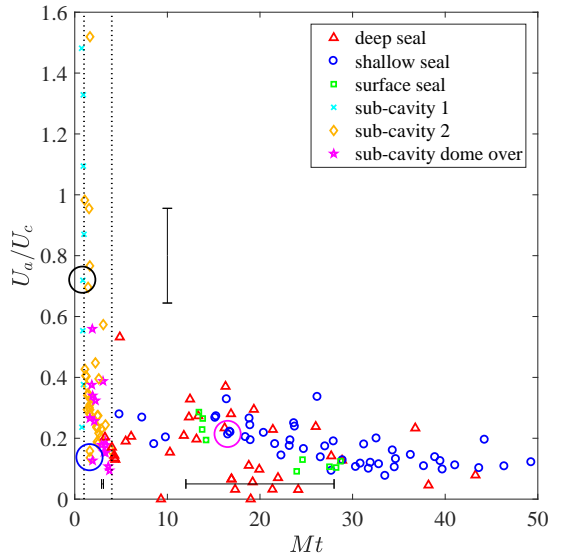

Figure 13: Downward cavity velocity of multi-droplet streams and jets. a) Depth of the cavity in time for four values of $M t$. The black line represents a multi-droplet stream with $\tilde{f}=0.14$ and for a case with sub-cavity 1 collapse. The blue curve represents a multi-droplet stream with sub-cavity 2 collapse and $\tilde{f}=0.26$. A linear fit is applied to estimate the average cavity velocity (red dashed line). The magenta line represents a multi-droplet stream with $\tilde{f}=0.27$, which displays shallow seal behavior. Note that as $M t$ increases, the oscillations in the cavity velocity become less pronounced. A jet (green line) also forms a shallow seal cavity with velocity nearly identical to the $M t=16.5$ mutli-droplet case. b) The average downward cavity velocity $\left(U_{c}\right)$ normalized by the stream velocity $\left(U_{s}\right)$ as a function of $\tilde{f}$. The black dashed line represents Eq. ?? and the red dotted line represents the theoretical velocity ratio for a jet. The mean velocity ratio for all 65 jet cases is marked by the grey dot and a standard box and whisker plot $(\tilde{f}=1)$. c) The amplitude of oscillation $\left(U_{a}\right)$ normalized by the average cavity velocity $\left(U_{c}\right)$ as a function of $M t$. The vertical dotted lines indicate transitional $M t$ numbers of 1 and 4 . The large circles in b) and c) outline data points represented by the corresponding colors of the lines in a). 
droplet impact by multiplying the left hand side by $\tilde{f}$

$$
\frac{1}{2} \rho\left(U_{s}-U_{c}\right)^{2} \tilde{f}=\frac{1}{2} \rho U_{c}^{2} .
$$

Now solving this for $U_{c} / U_{s}$ we get

$$
\frac{U_{c}}{U_{s}}=\frac{\sqrt{\tilde{f}}}{1+\sqrt{\tilde{f}}},
$$

where $\tilde{f} \in[0,1]$ (Fig. 3d). As shown in Fig. 13 , this modified theory accurately captures the experimentally measured cavity velocities. As $\tilde{f}$ approaches one, the multi-droplet stream becomes more jet-like approaching the theoretical value of $1 / 2$. Experimental values for all of the 65 jets studied have a mean value of 0.492 as marked. Most studies agree that the jet cavity velocity equals half of the jet velocity (Oguz et al., 1992, 1995; Zhu et al., 2000, Qu et al., 2013).

To describe the oscillations in the cavity we consider the black line in Fig. 13 , which represents a cavity formed by the impact of a multi-droplet stream with $M t=0.88$. Taking the numeric derivative of this curve we obtain the downward cavity velocity over time, which is found to be periodic over the time between impact of successive droplets (appearing similar to a sine wave). The oscillating amplitude of the cavity velocity is then averaged by finding the mean amplitudes of all periods $\left(U_{a}=\frac{1}{N} \sum_{n=1}^{N}\left(u_{n}^{\max }-u_{n}^{\min }\right) / 2\right.$, where $u_{n}^{\max }$ and $u_{n}^{\min }$ are the maximum and minimum velocities within the $n^{t h}$ period). Because $M t<1$

we can see the first sub-cavity reach its maximum size at $T_{\text {max }}$. The growth of a multi-droplet-stream cavity can be modeled by constructing a superposition of curves from portions of $h(t)$ vs. $t$, where each curve represents the growth of a sub-cavity. When $M t=1$, the second droplet impacts just as the curve reaches its apex and the parabolic shaped growth is repeated (Fig. $13 \mathrm{a}$ black line). When $M t=2$, the second droplet impacts at $T_{\max } / 2$ and only this first part of the curve is repeated (not labeled). Thus, the portion of the parabola that is repeated is from $t=0$ to $t=T_{\max } / M t$. When $M t>4$ the portion of the curve that is repeated is nearly a straight line and hence oscillations in the cavity velocity are small (Fig. 13 magenta line). Not only can this be seen in Fig. 13 but it can also be seen by comparing the murals of the sphere like cavities in Figs. 9a, 10a, and 12 a , against the uneven growth rate of cavities created by multi-droplet streams with low $M t$ as seen in Figs. 7 and 8 . Quantitatively this means that the average amplitude of oscillation of the cavity velocity, $U_{a}$, normalized by the average cavity velocity, $U_{c}$, depends on $M t$ (Fig. 13.). At $M t<4$ the cavity velocity becomes very unsteady, and around a $M t=1$ the amplitude of oscillation is on the order of the average cavity velocity.

\section{Cavity dimensions}

In this section we will discuss the various cavity dimensions including pinch-off depth $h_{p}$, the depth of the cavity bottom at pinch-off $h_{b}$, the cavity depth after 
the entire stream has impacted $h_{c}$, and the average cavity diameter $d_{c}$ as shown in Fig. 4 .

\subsection{Pinch-off depth}

The pinch-off depth $\left(h_{p}\right)$ is affected by the type of cavity closure, and thus depends on $M t, W e^{*}$ and $B o^{*}$. Fig. 14 plots the dimensionless pinch-off depth as a function of $M t$, illustrating this dependence on the closure regime. At $M t<$ 1 , when sub-cavity 1 collapse occurs the pinch-off depth is approximately equal to $d_{d}$ (non-modified droplet diameter). At $1<M t<4$ sub-cavity 2 collapse occurs and the non-dimensional pinch-off depth doubles to approximately $2 d_{d}$. In this same matryoshka number range, but at $W e^{*}>315$ sub-cavity dome over occurs. The depth of sub-cavity dome over is unpredictable and multiple sub-cavities may dome over within one matryoshka cavity. At $M t>4$ we see the three pinch-offs common to solid-sphere water entry (i.e., surface, shallow and deep seal). Surface seal occurs at the surface $h_{p} / h_{c}=0$. Shallow seal is on the order of the capillary length, approximately three to six droplet diameters $\left(3<h_{p} / d_{d}<6\right)$ below the surface and increasing slightly as $M t$ increases. Deep seal occurs approximately halfway between the surface and the bottom of the cavity $\left(h_{p} / h_{b} \approx 1 / 2\right)$.

In the case of deep seal, the ratio of pinch-off depth to cavity depth after the entire stream has impacted the cavity reveals a relatively constant ratio $h_{p} / h_{c} \approx 1 / 3$ (Fig. 14p), which is the same as reported by Oguz et al. (1995) for jets. Birkhoff \& Zarantonello (1957) showed theoretically that when the density of the jet and pool are equal, the depth of the cavity equals the length of the jet used to form it (i.e., $h_{c}=L_{j}$ ). We can use this to explain the ratio $h_{p} / h_{c}=1 / 3$. The jet is cut off when pinch-off occurs and the lower portion of the cavity contains a portion of the jet that is $\frac{1}{2} h_{b}$ long. This jet will add its remaining length to the depth of the cavity $\left(h_{c}=h_{b}+\frac{1}{2} h_{b}\right)$, thus, $h_{p}=\frac{1}{2} h_{b}=\frac{1}{3} h_{c}$. A similar relationship between $h_{p}, h_{b}$ and $h_{c}$ is derived for multi-droplet streams in $\S 5.3$.

\subsection{Predicting cavity depth and diameter using an energy balance}

Cavity dimensions can be predicted by an energy analysis similar to that performed by Engel (1966). The kinetic energy of a mulit-droplet stream of water can be expressed as

$$
K E_{d}=\frac{\pi}{12} \rho d_{d}^{3} U_{s}^{2} N
$$

where $N$ is the number of droplets that impact the cavity, and $U_{s}$ is the velocity of the stream. The jet kinetic energy can be expressed by

$$
K E_{j}=\frac{\pi}{8} \rho d_{j}^{2} L_{j} U_{s}^{2} .
$$


a)

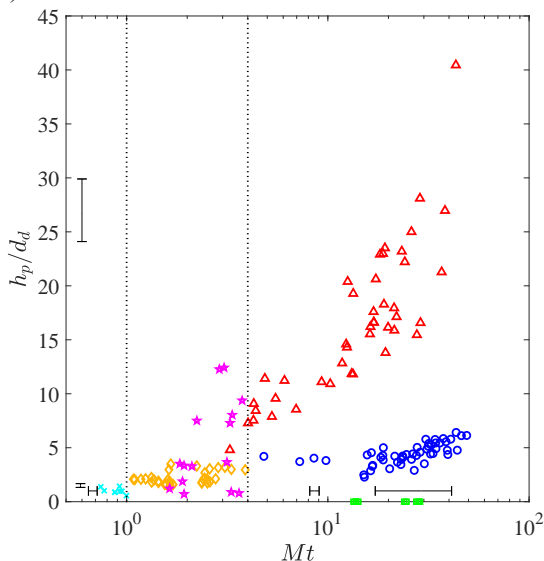

b)

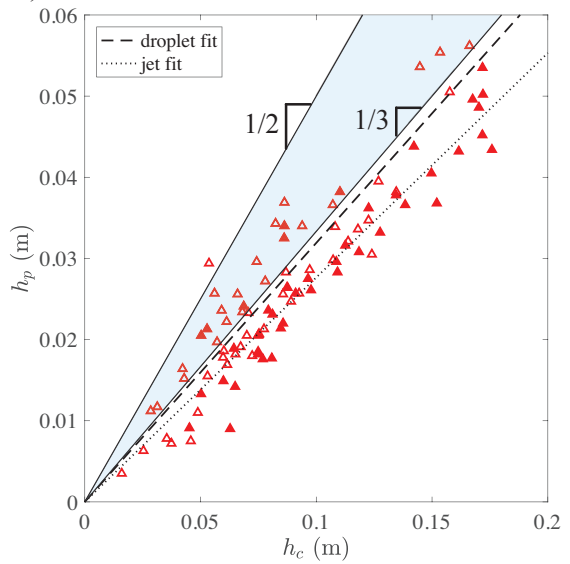

Figure 14: Pinch-off depth of multi-droplet streams. a) Non-dimensional pinchoff depth for all multi-droplet streams as a function of $M t$. Pinch-off depth is normalized by the non-modified droplet diameter $d_{d}$. b) Pinch-off depth as a function of cavity depth for the deep seal regime only for multi-droplet streams and jets. The upper bound for the theoretical range is $h_{p}=\frac{1}{2} h_{c}$, as predicted in \$5.3. The lower bound predicted for droplets is set by the line $h_{p}=\frac{1}{3} h_{c}$, which is also the theoretically predicted pinch-off depth for jets. The least squares fit to the droplet data has a slope of 0.319 and the fit to the jet data 0.277 . Symbols and data are the same as in Fig. 6 
In this study, the stream enters the cavity until the pinch-off event blocks further flow. Hence, $N$ can be calculated as

$$
N=f t_{p}-f h_{p} / U_{s},
$$

where $f t_{p}$ represents the number of droplets that pass the undisturbed free surface before $t_{p}$ and $f h_{p} / U_{s}$ represents the number of droplets that do not make it into the lower portion of the cavity. The jet length, $L_{j}$, can be similarly calculated as

$$
L_{j}=U_{s} t_{p}-h_{p} .
$$

The potential energy of the cavity, which is equal to the work done on the pool by the stream, can be broken up into two portions, the energy due to the hydrostatic pressure surrounding the cavity, $P E_{p r}$, and the energy due to the generated surface area, $P E_{\sigma}$. We model the maximum cavity size described in $\S 2$ as a long narrow cylinder of constant diameter, $d_{c}$, and depth $h_{c}$. The potential energy from pressure $\left(P E_{p r}=\int_{V} \rho g z d V\right)$ simplifies to

$$
P E_{p r}=\frac{\pi}{8} \rho g h_{c}^{2} d_{c}^{2} .
$$

The potential energy of the cavity due to generated surface area is

$$
P E_{\sigma}=\pi d_{c} h_{c} \sigma .
$$

Equating the kinetic (Eq. ??) to the potential energies (Eqs. ?? and ??) for a multi-droplet stream and rearranging yields

$$
\left(d_{c}^{2} \rho g\right) h_{c}^{2}+\left(8 d_{c} \sigma\right) h_{c}-\frac{2}{3} \rho d_{d}^{3} U_{s}^{2} N=0 .
$$

Assuming a positive solution to the quadratic formula, non-dimensionalizing and simplifying we find

$$
\frac{h_{c} d_{c}}{d_{d}^{2}}=-\frac{4}{B o}+\sqrt{\left(\frac{4}{B o}\right)^{2}+\frac{2 N}{3} F r} \equiv \Omega_{d},
$$

for droplets. Performing the same analysis for a jet yields

$$
\frac{h_{c} d_{c}}{d_{j}^{2}}=-\frac{4}{B o}+\sqrt{\left(\frac{4}{B o}\right)^{2}+\frac{L_{j}}{d_{j}} F r} \equiv \Omega_{j} .
$$

The left hand side of Eqs. ?? \& ?? can be thought of as a characteristic cavity size, which is a function of Bo and Fr. Experimental data in Fig. 15 shows good agreement with this relationship. Eqs. ?? \& ?? only tell us about the product of $h_{c}$ and $d_{c}$. If we want to predict the cavity depth $\left(h_{c}\right)$ we must know the cavity diameter $\left(d_{c}\right)$. However, the ratio $d_{c} / d_{j, d}$ was empirically shown to be a linear function of impact velocity (Fig. 5). 


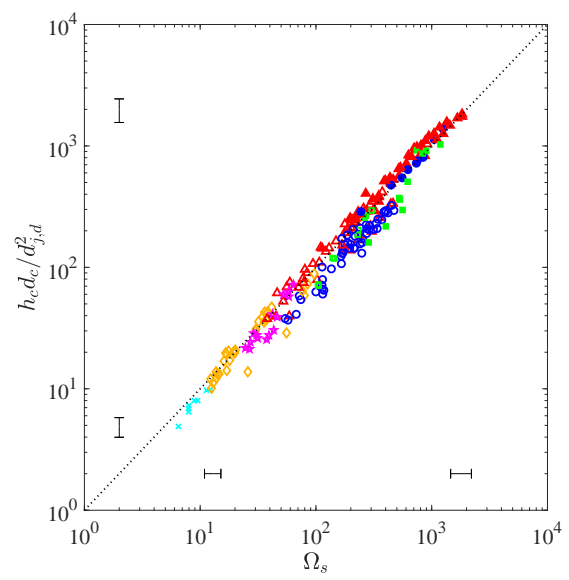

Figure 15: The non-dimensional cavity size for both jets and multi-droplet streams is collapsed by a function of $B o$ and $F r$ computed from an energy balance. The theoretical predictions of Eqs. ?? and ?? are shown by the dotted line, as both equations collapse to a single curve. Note that the droplet diameter used in this analysis is $d_{d}$, the non-modified droplet diameter. Symbols and data are the same as in Fig. 6 .

\subsection{Predicting cavity depth using the Bernoulli equation}

We will now discuss another way that the depth of the cavity, $h_{c}$, can be predicted. Recall that Birkhoff \& Zarantonello (1957) showed mathematically that when the density of the jet and the density of the pool are equal, then $h_{c}=L_{j}$. The ratio of the cavity depth to the jet length for a given time can be expressed as

$$
\frac{h(t)}{l_{j}(t)}=\frac{U_{c} t}{\left(U_{s}-U_{c}\right) t} .
$$

where $l_{j}(t)$ is the length of jet that has impacted the cavity by time $t$. By inserting $U_{c}=\frac{1}{2} U_{s}$ from Eq. ?? and simplifying we get:

$$
\frac{h(t)}{l_{j}(t)}=1 .
$$

We can apply the same analysis to a multi-droplet stream by solving for $U_{c}$ in Eq. ?? and placing it into Eq. ?? yielding

$$
\frac{h(t)}{l_{d}(t)}=\sqrt{\tilde{f}},
$$

where $l_{d}(t)$ is approximately equal to the distance from the first droplet that impacts to the last droplet that has impacted by time $t$. These equations work well to predict the depth of the cavity, $h_{c}$ for jets in Fig. 16 and droplets in 
a)

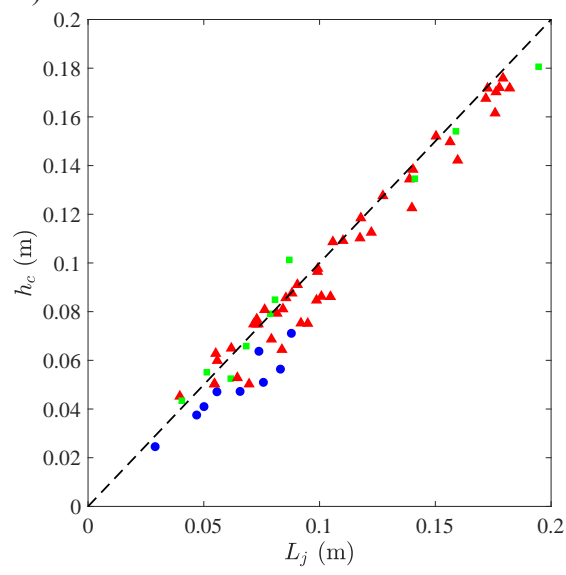

b)

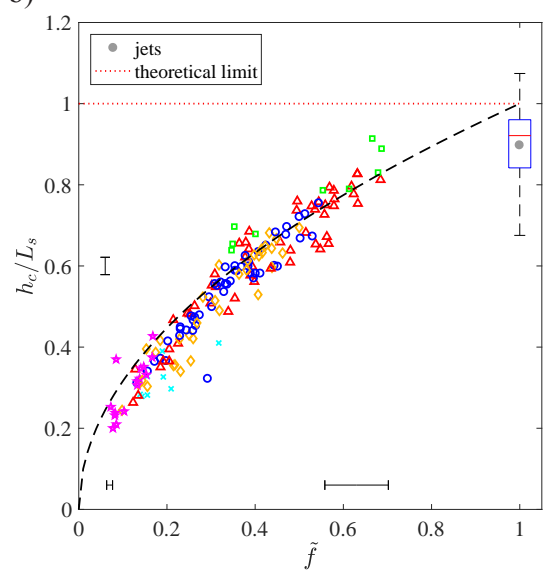

Figure 16: a) The length of the jet that impacts the cavity bottom equals the depth of the cavity that it creates. b) An equivalent expression for multi-droplet streams works to predict the depth of the cavity, $h_{c}$. The dashed lines in a and b represent Eqs. ?? and ?? respectively. The gray circle in b) represents the average $h_{c} / L_{j}$ for all of the jet cases with the uncertainty bands at two standard deviations. $L_{s}$ represents the length of either the multi-droplet stream or jet stream. Symbols and data are the same as in Fig. 6.

Fig. 16p. Eq. ?? slightly over predicts the depth of the cavity for jets. This can be explained by recalling that in Eq. ?? the effect of gravity was neglected. Thus, the hydrostatic pressure, which pushes against the impinging jet is not taken into account; therefore, departure from Eq. ?? increases with increasing depth. One could modify these equations to include gravity but the overall conclusion remains the same.

We can now revisit the relationship between the cavity depths $h_{p}$ and $h_{c}$. The total cavity depth for a multi-droplet stream can be written as $h_{c}=h_{b}+\frac{1}{2} h_{b} \sqrt{\tilde{f}}$ leading to $h_{p}=\frac{1}{2} h_{b}=h_{c} /(\sqrt{\tilde{f}}+2)$ where $h_{p} \in\left[\frac{1}{3} h_{c}, \frac{1}{2} h_{c}\right]$ which is closely supported by the trends of Fig. $14 \mathrm{~b}$. It is likely that the difference between the theoretical estimate and the experimental result is that in the experiment it is possible for a portion of the jet or a droplet to get 'caught' in the pinch-off event, and not descend downward with the rest of the fluid, thus reducing the effective full stream cavity depth.

Finally, the depth of the cavity can be estimated using Eq. ?? by substituting $h_{c}$ for $L_{j}$ and assuming a semi-infinite jet that is cut short by the pinch-off event

$$
h_{c}=U_{s} t_{p}-h_{p}
$$

We can then replace $h_{p}$ depending on the cavity type: for deep seal we use $h_{p}=\frac{1}{3} h_{c}$, for shallow seal $h_{p}$ is approximately equal to the capillary length $(\lambda)$ 
and for surface seal $h_{p}=0$, thus each of these cases becomes

$$
h_{c, j e t s}= \begin{cases}\frac{3}{4} U_{s} t_{p} & \text { deep } \\ U_{s} t_{p}-\lambda & \text { shallow } \\ U_{s} t_{p} & \text { surface }\end{cases}
$$

Applying this same method to multi-droplet streams, but using $L_{d}=\frac{h_{c}}{\sqrt{\tilde{f}}}$, the equations for deep, shallow, and surface seal become

$$
h_{c, \text { droplets }}= \begin{cases}\frac{U_{s} t_{p} \sqrt{\tilde{f}}}{1+\frac{1}{3} \sqrt{\tilde{f}}} & \text { deep } \\ \left(U_{s} t_{p}-\lambda\right) \sqrt{\tilde{f}} & \text { shallow } \\ U_{s} t_{p} \sqrt{\tilde{f}} & \text { surface. }\end{cases}
$$

These analyses shows that the dimensions of the cavity for jets can be used to unravel the multi-droplet cavity dimensions and that the two different stream types are indeed related physically. For instance, if $\tilde{f}=1$, which is the case for a jet, Eqs. ?? simplify to the jet equations Eqs. ??.

We emphasize the differences between the models presented in $\$ 5.2 \& 5.3$ The energy balance based model in $\$ 5.2$ predicts the cavity size (a non-dimensional measurement including both cavity depth $\left(h_{c}\right)$ and cavity diameter $\left.\left(d_{c}\right)\right)$ by considering all of the kinetic energy of the incoming stream and comparing it to the potential energy of the entire cavity that it forms. In order to predict cavity size $h_{c} d_{c} / d_{s}^{2}$ and obtain some information on the cavity diameter $d_{c}$ surface tension and gravity must be considered as both resisting the radial expansion of the cavity and contributing to the cavity's potential energy. If one desires to obtain the cavity depth using this energy balance based model the average cavity diameter $d_{c}$ must be known. On the other hand the Bernoulli based approach in $\$ 5.3$ predicts $h_{c}$ directly by using an energy balance solely at the cavity bottom. Surface tension and gravitational parameters are unnecessary in this method because the energy balance occurs on a short section of a single streamline (the axis of the flow), where the effect of these parameters is negligible. To compare the fidelity of these two methods in predicting cavity depth we plot measured verses predicted values of $h_{c}$ in Fig. 17. The energy based method predicts $h_{c}=\Omega_{s} d_{s}^{2} / d_{c}$ (Eqs. ?? \& ?? rearranged), where $d_{c}$ is evaluated from experimental measurements (Fig. 4p). The Bernoulli method predicts $h_{c}=l_{s} \sqrt{\tilde{f}}$ (Eqs. ?? \& ?? rearranged). The Bernoulli method results in a slightly more accurate prediction of $h_{c}$ without considering surface tension and gravity. In fact, introducing gravity and surface tension does not improve the model; implying that the surface tension and gravity may not be dominant contributors to the downward growth of the cavity. However, they are important when considering the radial growth and collapse of the cavity as outlined in section $\S 3$. 


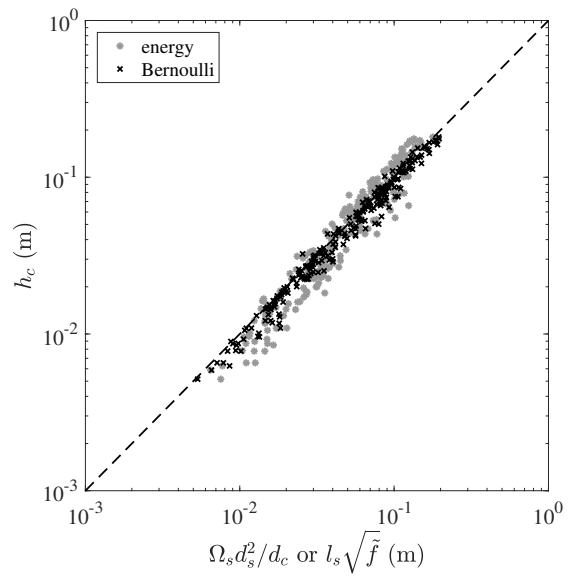

Figure 17: A comparison of the two methods for predicting cavity depth $h_{c}$ is shown. The y-axis is the measured value of the cavity depth $h_{c}$, while the $\mathrm{x}$-axis is the predicted value of $h_{c}$ based on two different approaches. The first term on the $\mathrm{x}$-axis comes from the the energy based method $\left(h_{c}=\Omega_{s} d_{s}^{2} / d_{c}\right.$, Eqs. ?? \& ?? rearranged), while the second term is from the Bernoulli based method $\left(h_{c}=l_{s} \sqrt{\tilde{f}}\right.$, Eqs. ?? \& ?? rearranged) as marked in the legend. An exact prediction is represented by the dashed line.

\section{Conclusion}

Many past water impact studies have focused separately on the initial impact of single droplets, jets or solids into a deep pool of liquid. Some groups have loosely connected multi-droplet stream impact to jet and solid sphere impact (Hurd et al. 2015, Bouwhuis et al. 2016), yet have not necessarily studied an expansive range of parameters nor threaded the underlying physical scaling laws together quantitatively. Despite the fact that the initial impact of multi-droplet streams create different cavity aesthetics (rough ridges) when compared to the cavities made by jets, or solid hydrophobic spheres (Fig. 1), our experimental work shows that all initial impact types create intrinsically similar cavities that can be connected by physical arguments.

Empirically, we show that the diameter of a cavity made by a multi-droplet stream matches that made by a jet if the diameter of the droplets are 1.75 times the jet diameter. This modification in diameter is valid over a large range of impact parameters. The cavity types can be separated by introducing a new dimensionless number we coin the matryoshka number, $M t$. When $M t<4$ sub-cavities behave independently from the cavity as a whole, forming cavities reminiscent of single droplet impact. In this region we find three new cavity shapes: subcavity 1 collapse, subcavity 2 collapse and subcavity dome over. When $M t>4$ the multi-droplet streams act like jets creating well known cavities 
similar to ones created by hydrophobic spheres and distinguished by their pinchoff locations: deep seal, shallow seal and surface seal. Further, the cavities of multi-droplet streams become more smooth, jet-like, and approach the jet cavity velocity and depth as the dimensionless droplet stream frequency $\tilde{f} \rightarrow 1$. Each of the regimes can also be accurately predicted by the $B o^{*}$ and $W e^{*}$ numbers as one might expect. Finally, the cavity dimensions (i.e., pinch-off depth, cavity depth and cavity diameter) can be predicted from a simple energy balance or by expanding the work of Birkhoff \& Zarantonello (1957) on jets.

The scope of future work might include the effect of increasing jet and droplet sizes (if possible), predicting when each sub-cavity will dome over and finding a theoretical explanation for the scaling of the cavity diameter between multidroplet streams and jets (e.g., 1.75 found in Fig. 5). There is also the question of continuous impact of the jet or multi-droplet stream, and how the underwater bubble field will interact with the incoming stream and whether similar cavities can be formed after the initial impact and collapse of the first cavity. One application of these findings might be the impact of multiple objects and object types in succession upon the water surface to influence the cavity shape and resultant forces.

\section{Acknowledgments}

N.B.S., T.T.T. and J.B. acknowledge funding from the Office of Naval Research, Navy Undersea Research Program (grant N0001414WX00811), monitored by Ms. Maria Medeiros. J.B. acknowledges funding from the Naval Undersea Warfare Center In-House Laboratory Independent Research program, monitored by Mr. Neil Dubois. N.B.S and T.T.T acknowledge funding from the Utah State University Research and Graduate Studies Development Grant Program. We gratefully acknowledge Randy Hurd as a helpful editor, reader and discussions starter and for coining the term matryoshka number.

\section{A Alternative scaling}

Bouwhuis et al. (2016) studied multi-droplet stream impact on a pool using boundary integral simulations with some experimental data for validation. They studied the shallow seal regime for micrometer-sized droplets with $B o \sim O\left(10^{-4}\right)$ and developed scaling arguments using a modified Weber number defined by the cavity velocity, $W e_{m}=\rho U_{c}^{2} d_{d} / \sigma$, to predict $d_{c}$ and $t_{p}$. To verify the validity of these trends over a more expansive parameter range we plot our experimental data for their proposed scaling arguments. Fig. 18 shows that the scaling of $d_{c}$ and $t_{p}$ works for the shallow seal data in each case, but does not work well for the other pinch-off regimes. The dimensionless cavity diameter was better predicted by plotting it against impact velocity as shown in Fig. 5 and the nondimensional pinch-off time is better predicted with $W e^{*}$ as shown in Fig. 11 . 

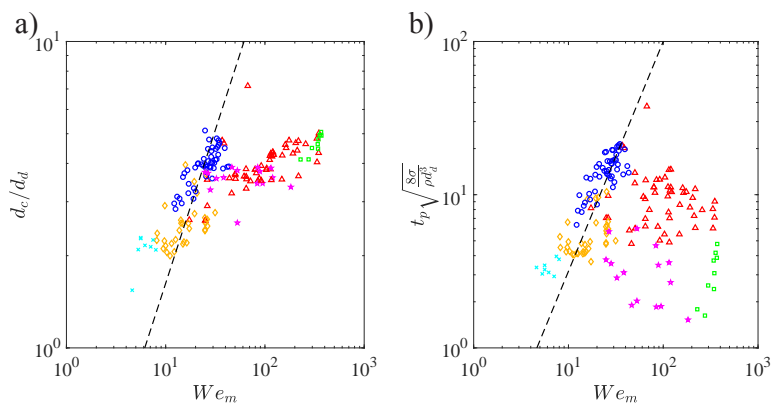

Figure 18: Prediction of $d_{c}$ and $t_{p}$ with a modified Weber number, $W e_{m}=$ $\rho U_{c}^{2} d_{d} / \sigma$, as discussed by Bouwhuis et al. (2016). a) The nondimensional cavity diameter is said to scale as $d_{c} / d_{d}=0.1625 W e_{m}$ shown by the dashed line. $\mathrm{b}$ ) The pinch-off time nondimensionalized by a capillary time scale is said to scale with $W e_{m}^{3 / 2}$ with the dashed line showing $t_{p} \sqrt{8 \sigma / \rho d_{d}^{3}}=0.1 W e_{m}^{3 / 2}$. Symbols are outlined in the legend of Fig. 6a.

\section{References}

Aristoff, Jeffrey M. \& Bush, John W. M. 2009 Water entry of small hydrophobic spheres. Journal of Fluid Mechanics 619, 45-78.

Bick, Alexander G., Ristenpart, William D., van Nierop, Ernst A. \& Stone, Howard A. 2010 Bubble formation via multidrop impacts. Physics of Fluids 22 (4).

Birkhoff, G \& Zarantonello, EH 1957 Jets, Wakes, and Cavities (Series in Applied Mathematics and Mechanics, vol. 2). Academic Press, New York.

Bouwhuis, Wilco, Huang, Xin, Chan, Chon U, Frommhold, Philipp E., Ohl, Claus-Dieter, Lohse, Detlef, Snoeijer, Jacco H. \& VAN DER MeEr, Devaraj 2016 Impact of a high-speed train of microdrops on a liquid pool. Journal of Fluid Mechanics 792, 850-868.

Cole, David 2007 The splashing morphology of liquid-liquid impacts. PhD thesis, James Cook University.

Coleman, Hugh W \& Steele, W Glenn 2009 Experimentation, validation, and uncertainty analysis for engineers. John Wiley \& Sons.

Duclaux, V., Caillé, F., Duez, C., Ybert, C., Bocquet, L. \& Clanet, C. 2007 Dynamics of transient cavities. Journal of Fluid Mechanics 591, 119.

Engel, Olive G. 1966 Crater depth in fluid impacts. Journal of Applied Physics 37 (4), 1798-1808. 
Engel, Olive G. 1967 Initial pressure, initial flow velocity, and the time dependence of crater depth in fluid impacts. Journal of Applied Physics 38 (10), $3935-3940$.

Franz, G. J. 1959 Splashes as sources of sound in liquids. The Journal of the Acoustical Society of America 31 (8), 1080-1096.

Hurd, R., Fanning, T., Pan, Z., Mabey, C., Bodily, K., Hacking, K., Speirs, N. \& Truscott, T. 2015 Matryoshka cavity. Physics of Fluids 27 (9).

Kersten, B., Ohl, C. D. \& Prosperetti, A. 2003 Transient impact of a liquid column on a miscible liquid surface. Physics of Fluids 15 (3), 821-824.

Kiger, Kenneth T. \& Duncan, James H. 2012 Air-entrainment mechanisms in plunging jets and breaking waves. Annual Review of Fluid Mechanics 44 (1), 563-596, arXiv: http://dx.doi.org/10.1146/annurev-fluid-122109160724 .

Leng, Liow Jong 2001 Splash formation by spherical drops. Journal of Fluid Mechanics 427, 73-105.

Lin, SP \& REITZ, RD 1998 Drop and spray formation from a liquid jet. Annual Review of Fluid Mechanics 30 (1), 85-105.

Lorenceau, Élise, Quéré, David \& Eggers, Jens 2004 Air entrainment by a viscous jet plunging into a bath. Phys. Rev. Lett. 93, 254501.

Mansoor, M. M., Marston, J. O., Vakarelski, I. U. \& Thoroddsen, S. T. 2014 Water entry without surface seal: extended cavity formation. Journal of Fluid Mechanics 743, 295-326.

Marston, J. O., Truscott, T. T., Speirs, N. B., Mansoor, M. M. \& Thoroddsen, S. T. 2016 Crown sealing and buckling instability during water entry of spheres. Journal of Fluid Mechanics 794, 506-529.

May, Albert \& Woodhull, Jean C. 1948 Drag coefficients of steel spheres entering water vertically. Journal of Applied Physics 19 (12), 1109-1121.

Morton, David, Rudman, Murray \& Jong-Leng, Liow 2000 An investigation of the flow regimes resulting from splashing drops. Physics of Fluids 12 (4), 747-763, arXiv: http://dx.doi.org/10.1063/1.870332.

Oguz, Hasan N. \& Prosperetti, Andrea 1990 Bubble entrainment by the impact of drops on liquid surfaces. Journal of Fluid Mechanics 219, 143-179.

Oguz, Hasan N., Prosperetti, Andrea \& Kolaini, Ali R. 1995 Air entrapment by a falling water mass. Journal of Fluid Mechanics 294, 181207. 
Oguz, H.N., Prosperetti, A. \& Lezzi, A. M. 1992 Examples of airentraining flows. Physics of Fluids A 4 (4), 649-651.

Qu, Xiaoliang, Goharzadeh, Afshin, Khezzar, Lyes \& Molki, ArMAN 2013 Experimental characterization of air-entrainment in a plunging jet. Experimental Thermal and Fluid Science 44, 51 - 61.

Ray, Bahni, Biswas, Gautam \& Sharma, Ashutosh 2015 Regimes during liquid drop impact on a liquid pool. Journal of Fluid Mechanics 768, 492-523.

Rodriguez, Francisco \& Mesler, Russell 1988 The penetration of dropformed vortex rings into pools of liquid. Journal of Colloid and Interface Science 121 (1), 121 - 129.

Soh, Wee King, Khoo, Boo Cheong \& Yuen, W. Y. Daniel 2005 The entrainment of air by water jet impinging on a free surface. Experiments in Fluids 39 (3), 498-506.

Thoroddsen, S. T., Eтoh, T. G. \& Takehara, K. 2003 Air entrapment under an impacting drop. Journal of Fluid Mechanics 478, 125-134.

Truscott, Tadd T., Epps, Brenden P. \& Belden, Jesse 2014 Water entry of projectiles. Annual Review of Fluid Mechanics 46 (1), 355-378, arXiv: http://dx.doi.org/10.1146/annurev-fluid-011212-140753.

Truscott, Tadd T., Epps, Brenden P. \& Techet, Alexandra H. 2012 Unsteady forces on spheres during free-surface water entry. Journal of Fluid Mechanics 704, 173-210.

Worthington, A. M. \& Cole, R. S. 1900 Impact with a liquid surface studied by the aid of instantaneous photography. Philosophical Transactions of the Royal Society of London. Series A, Containing Papers of a Mathematical or Physical Character 194, 175-199.

YARIN, A.L. 2006 Drop impact dynamics: splashing, spreading, receding, bouncing. Annu. Rev. Fluid Mech. 38, 159-192.

Zhu, Yonggang, OĞuz, Hasan N. \& Prosperetti, Andrea 2000 On the mechanism of air entrainment by liquid jets at a free surface. Journal of Fluid Mechanics 404, 151-177. 